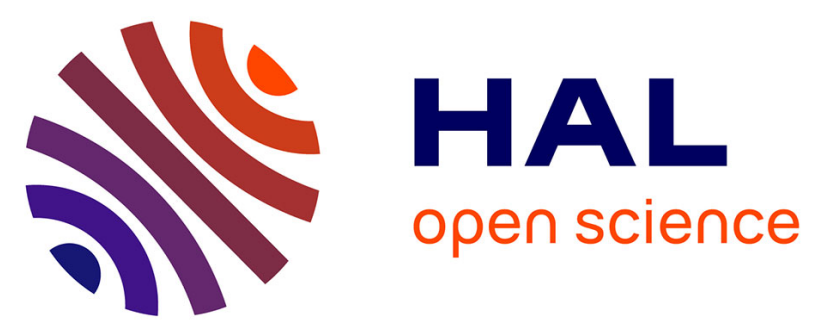

\title{
All at Once! A Comprehensive and Tractable Semi-Parametric Method to Elicit Prospect Theory Components
}

Yao Thibaut Kpegli, Brice Corgnet, Adam Zylbersztejn

\section{To cite this version:}

Yao Thibaut Kpegli, Brice Corgnet, Adam Zylbersztejn. All at Once! A Comprehensive and Tractable Semi-Parametric Method to Elicit Prospect Theory Components. 2020. halshs-03016517

\section{HAL Id: halshs-03016517 https://shs.hal.science/halshs-03016517}

Preprint submitted on 20 Nov 2020

HAL is a multi-disciplinary open access archive for the deposit and dissemination of scientific research documents, whether they are published or not. The documents may come from teaching and research institutions in France or abroad, or from public or private research centers.
L'archive ouverte pluridisciplinaire HAL, est destinée au dépôt et à la diffusion de documents scientifiques de niveau recherche, publiés ou non, émanant des établissements d'enseignement et de recherche français ou étrangers, des laboratoires publics ou privés. 
UMR 5824

93. chemin des Mouilles 69130 Ecully - France

Maison de l'Universite, Bâtiment B 10, rue Trefilerie 42023 Saint-Etienne cedex 02 - France http://www.gate.cnrs.fr gate@gate.cnrs.fr

\title{
All at Once! A Comprehensive and Tractable Semi- Parametric Method to Elicit Prospect Theory Components
}

\author{
Yao Thibaut Kpegli, Brice Corgnet, Adam Zylbersztejn
}

\begin{abstract}
:
Eliciting all the components of prospect theory -curvature of the utility function, weighting function and loss aversion- remains an open empirical challenge. We develop a semi-parametric method that keeps the tractability of parametric methods while providing more precise estimates. Using the data of Tversky and Kahneman (1992), we revisit their main parametric results. We reject the convexity of the utility function in the loss domain, find lower probability weighting, and confirm loss aversion. We also report that the probability weighting function does not exhibit duality and equality across domains, in line with cumulative prospect theory and in contrast with original prospect and rank dependent utility theories.
\end{abstract}

\section{Keywords:}

Prospect theory, semi-parametric estimation, risk attitudes, weighting function, loss aversion

JEL codes:

D81, C91 


\title{
All at Once! A Comprehensive and Tractable Semi-Parametric Method to Elicit Prospect Theory Components*
}

\author{
Yao Thibaut Kpegli ${ }^{\dagger} \quad$ Brice Corgnet ${ }^{\ddagger} \quad$ Adam Zylbersztejn ${ }^{\S}$
}

November 17, 2020

\begin{abstract}
Eliciting all the components of prospect theory -curvature of the utility function, weighting function and loss aversion- remains an open empirical challenge. We develop a semi-parametric method that keeps the tractability of parametric methods while providing more precise estimates. Using the data of Tversky and Kahneman (1992), we revisit their main parametric results. We reject the convexity of the utility function in the loss domain, find lower probability weighting, and confirm loss aversion. We also report that the probability weighting function does not exhibit duality and equality across domains, in line with cumulative prospect theory and in contrast with original prospect and rank dependent utility theories.
\end{abstract}

Keywords: Prospect theory; semi-parametric estimation; risk attitudes; weighting function, loss aversion.

JEL codes : D81, C91

\section{Introduction}

There is now a large body of empirical evidence showing systematic violations of expected utility theory (EUT; see Starmer, 2000, for a review). The original version of Prospect Theory (OPT; see Kahneman and Tversky, 1979, henceforth KT79) and its subsequent refinements, most notably Cumulative Prospect Theory (CPT; see Tversky and Kahneman, 1992, henceforth KT92), explain these empirical violations by introducing probability distortions (Bleichrodt and Pinto, 2000) and loss aversion (Wakker, 2010). Classical methods for eliciting risk attitudes (such as Holt and Laury, 2002) cannot account for these violations because they rely on EUT. New approaches have thus been proposed that build on CPT instead of EUT (Abdellaoui et al., 2008, henceforth ABL). Our focus here is on comprehensive methods that allow for an

\footnotetext{
${ }^{*}$ We gratefully acknowledge helpful comments from Peter Wakker, Fréderic Jouneau-Sion, as well as seminar participants at GATE Lyon-Saint-Etienne. We thank Richard Gonzalez and George Wu for sharing their dataset with us. This research has been performed within the framework of LABEX CORTEX (ANR-11-LABX-0042) of Université de Lyon, within the program Investissements d'Avenir (ANR-11-IDEX-007) run by the French National Research Agency (ANR).

†Univ Lyon, Université Lyon 2, GATE UMR 5824, F-69130 Ecully, France. Email: kpegli@gate.cnrs.fr

${ }_{\ddagger}^{\ddagger}$ Univ Lyon, Emlyon Business School, GATE UMR 5824, F-69130 Ecully, France. Email: corgnet@emlyon.com

${ }^{\S}$ Univ Lyon, Université Lyon 2, GATE UMR 5824, F-69130 Ecully, France. Email: zylbersztejn@gate.cnrs.fr
} 
estimation of all three components of individual risk and ambiguity attitudes: utility function, loss aversion and weighting function. These methods fall into three broad categories: parametric (parametric forms for utility and probability weighting functions), semi-parametric (parametric form for the utility function but not for the probability weighting function) and non-parametric (no parametric form on either function).

Parametric methods have four desirable properties that make them a popular choice in applied research (ABL). First, they are tractable because risk attitudes can be easily estimated through standard econometric tools such as ordinary and nonlinear least squares (Abdellaoui et al., 2007b). Second, they are efficient because they require relatively few individual observations, thus facilitating data collection (ABL). ${ }^{1}$ Third, they are easy (not cognitively demanding) for subjects to comprehend since they are based on simple choices (ABL). Finally, they are error-robust because they account for the fact that subjects can make mistakes by including error terms in the statistical estimations (TK92; ABL; Fehr-Duda et al., 2006; Gonzalez and Wu, 1999, henceforth GW99).

These virtues, however, come with costs. First, the estimation results are sensitive to the choice of the functional forms for the utility and probability weighting functions (Abdellaoui, 2000). ${ }^{2}$ Second, parametric methods only provide an overall measure of the goodness of fit of the model, rather than separate measures for each of the components of the model such as the utility and the probability weighting functions (see GW99).

None of the existing comprehensive methods, whether non-parametric or semi-parametric, possess the four desirable properties of parametric methods. ${ }^{3}$ Our goal is to fill this gap in the literature by developing a comprehensive semi-parametric method that possesses these desirable properties. Our method also comes with additional advantages. Since it does not require any specific conditions on the event space, it can be applied to various real-life uncertainty situations (Baillon et al., 2018; Van De Kuilen and Wakker, 2011). In addition, although we introduce our method under CPT, it can also be implemented under OPT and alternative theories such as rank dependent utility theory (henceforth RDU; see Quiggin, 1982; Gilboa, 1987; Schmeidler, 1989). It also circumvents important methodological issues previously raised in the literature, such as the arbitrary measurement of loss aversion (Wakker, 2010). Finally, from an experimental standpoint, our method allows for controlling the lower and the upper bound of indifferences values, thus facilitating the elicitation of risk attitudes. Indifference values can thus be elicited on a bounded interval in which case the implementation of the method relies on simpler choices relative to the case in which the interval is unbounded (Abdellaoui et al., 2007a; Wakker and Deneffe, 1996). In particular, our method can be implemented using the standard "switching outcome" procedure for eliciting certainty equivalents (GW99; Holt and Laury, 2002). ${ }^{4}$

This paper proceeds as follows. Section 2 outlines the existing semi-parametric and nonparametric methods. Section 3 presents our elicitation method under cumulative prospect theory. Section 4 provides an application of the method. We discuss our findings in Section 5. Section 6 concludes.

\footnotetext{
${ }^{1}$ Note that here the term efficiency only relates to the physical resources (such as time and money) used for data collection, and not the statistical properties (i.e., minimal variance) of a parametric estimator.

${ }^{2}$ For a review of parametric assumptions underlying standard structural estimations techniques, see Harrison and Rutström $(2008,2009)$

${ }^{3} \mathrm{ABL}$ can be considered as a notable exception (see Section 2 for a review). However, their estimation of the probability weighting function is restricted to only one specific probability weight. In contrast, our method allows for estimating the probability weighting function over any set of probabilities.

${ }^{4}$ The switching outcome procedure allows for inferring indifference values through a list of equally spaced certain outcomes ranging from the lower bound of the indifference value to the upper bound of the indifference value. This procedure requires knowing the upper and lower bounds of indifferences values (Booij and Van de Kuilen, 2009).
} 


\section{Existing semi-parametric and non-parametric meth- ods}

In this section, we review the existing semi- and non-parametric methods of eliciting risk attitudes under CPT. Table 1 provides an overview of these methods along the four desirable properties of parametric methods (tractability, efficiency, easiness, error-robustness) as well as comprehensiveness.

These methods typically use the tradeoff approach of Wakker and Deneffe (1996) in three steps. ${ }^{5}$ The popularity of the tradeoff method is mostly due to the fact that the elicitation of the utility function is robust to probability distortions. In the first step, the tradeoff method estimates the utility function by eliciting a sequence of outcomes $\left(x_{0}, x_{1}, x_{2}, \ldots, x_{n}\right)$ which are equally spaced in terms of utility: $u\left(x_{i}\right)-u\left(x_{i-1}\right)=$ constant for $i=1,2, \ldots, n$. In the second step, the utility function obtained in the first step is used to estimate the weighting function. If the utility function in the gain and loss domains is elicited on the same scale (Abdellaoui et al., 2007a, 2016), the loss aversion can then be inferred in a third step. The tradeoff method is not error-robust because it assumes that the first-step values $\left(x_{1}, x_{2}, \ldots, x_{n}\right)$ are elicited without errors. This assumption is particularly restrictive because the elicitation of these values is subject to error propagation. Indeed, in the first step of the tradeoff method, any error arising in the elicitation of a given value $\left(x_{i}\right)$ will hinder the estimation of subsequent values $\left(x_{i+1}, \ldots, x_{n}\right) .{ }^{6}$ It follows that semi-parametric and non-parametric elicitation techniques that rely on the tradeoff method are not error-robust (see Table 1). Moreover, ABL note that the complexity of the elicitation procedure makes the tradeoff method cognitively demanding. ${ }^{7}$

In the literature, three notable exceptions use alternative elicitation procedures to the tradeoff method (GW99; ABL; Abdellaoui et al., 2011a). ${ }^{8}$ However, GW99 is not a comprehensive method because it does not elicit loss aversion. In addition, Van De Kuilen and Wakker (2011) point to the lack of tractability and efficiency of this method. The semi-parametric method of ABL, in turn, satisfies the four appealing properties of parametric methods and allows researchers to assess the goodness of fit of the functional form used to estimate the utility function. Yet, ABL is not a comprehensive method because it estimates a specific probability weight instead of the whole range. Achieving comprehensiveness by including an additional step to estimate the probability weighting function, as in Abdellaoui et al. (2011a), comes at the cost of potentially multiplying response errors (Etchart-Vincent, 2004).

\footnotetext{
${ }^{5}$ See Abdellaoui (2000); Bleichrodt and Pinto (2000); Etchart-Vincent (2004, 2009a); Van De Kuilen and Wakker (2011); Booij and Van de Kuilen (2009); Abdellaoui et al. (2007a, 2016); Attema et al. (2018); Bleichrodt et al. (2018).

${ }^{6}$ Another issue with the standard tradeoff method is strategic responding (Harrison and Rutström, 2008; Abdellaoui et al., 2020). For the sake of illustration, suppose that the researcher is looking for outcomes $x_{1}$ and $x_{2}$ by eliciting a pair of chained indifference values $x_{1}$ and $x_{2}$ such that $\left(x_{1}, \$ 1 ; 0.5,0.5\right) \sim(\$ 10, \$ 5 ; 0.5,0.5)$ and $\left(x_{2}, \$ 1 ; 0.5,0.5\right) \sim\left(x_{1}, \$ 5 ; 0.5,0.5\right)$. One of these four lotteries is picked at random for payoff at the end of the experiment. Hence, the decision-making problem boils down to calibrating the following lottery: $R\left(x_{1}, x_{2}\right)=\left(\$ 1, \$ 5, \$ 10, x_{1}, x_{2} ; 1 / 4,1 / 4,1 / 8,1 / 4,1 / 8\right)$. This, in turn, provides incentives to overstate the values of $x_{1}$ and $x_{2}$, since $x_{1}^{*}>x_{1}$ and $x_{2}^{*}>x_{2}$ yields a lottery $R\left(x_{1}^{*}, x_{2}^{*}\right)$ that first-order stochastically dominates $R\left(x_{1}, x_{2}\right)$. Recently, Johnson et al. (2019) have developed a new mechanism, called PRINCE, that alleviates the issue by ex ante fixing the real choice situation that will be used to pay the subject at the end of the experiment.

${ }^{7}$ In the tradeoff method subjects always compare two binary lotteries, while methods that elicit certainty equivalents ask subjects to compare a certain amount with a binary lottery. Thus, the tradeoff method requires processing more information and is thus considered to be more cognitively demanding.

${ }^{8}$ Related method to Abdellaoui et al. (2011a) is the source method of Abdellaoui et al. (2011c) that allows to elicit the source function and utility function under biseparable preference model of Ghirardato and Marinacci (2001) with additional assumption that decision maker can assign subjective probabilities (i.e., beliefs) to events even when he doesn't maximize subjective expected utility. Another exception is the method of Bertani et al. (2019) to approximate probability weighting function. This method is very restrictive since it is valid only for dual theory of Yaari (1987) where utility is assumed to be linear.
} 
In addition, the approach used in both ABL and Abdellaoui et al. (2011a) suffers from two caveats when it comes to measuring loss aversion. First, estimating the utility function separately in the gain and loss domains makes it impossible to impose partial reflection (i.e., identical utility curvature in the gain and loss domains) which is often required to circumvent the arbitrary measurement of loss aversion. Indeed, Wakker (2010, pp. 267-270) ${ }^{9}$ shows that, when using power utility function, one can obtain any loss aversion parameter by rescaling the data when partial reflection is not imposed. ${ }^{10}$ By allowing for a joint estimation of the utility function in both domains, our method allows the researcher to test and impose partial refection whenever needed. ${ }^{11}$ Second, unlike our method, the approach of ABL and Abdellaoui et al. (2011a) does not constrain the lower and upper bounds of indifferences values when measuring loss aversion. This, in turn, precludes the use of the standard switching outcome procedure (Booij and Van de Kuilen, 2009) and makes the elicitation procedure more cognitively demanding (Abdellaoui et al., 2007a; Wakker and Deneffe, 1996).

\section{Method}

\subsection{Notations}

Consider a binary lottery $L=(x, y ; p, 1-p)$ yielding outcome $x$ with probability $p$ and outcome $y$ with probability $1-p$, both outcomes being real numbers. ${ }^{12}$ For notational convenience, let $x>y \geq 0(x<y \leq 0)$ for non-mixed prospects involving only gains (losses). For mixed prospects (i.e., involving both gains and losses), outcomes are denoted with an asterisk and $y^{*}<0<x^{*}$. $\succsim$ is a preference relation over prospects with $\succ(\sim)$ denoting strict preference (indifference). Preferences are represented by CPT with a probability weighting function $w^{i}$ and a value function $v$ as defined in equation (1) for non-mixed prospects and in equation (2) for the mixed ones:

$$
\begin{gathered}
C P T(L)=(v(x)-v(y)) w^{i}(p)+v(y) \\
C P T(L)=w^{+}(p) v(x)+w^{-}(1-p) v(y)
\end{gathered}
$$

where $w^{i}$ and $v$ are both continuous, strictly increasing and satisfying $v(0)=0, w^{i}(0)=0$ and $w^{i}(1)=1$, and $i=$ "+" ( $i=$ "-") stands for the gain (loss) domain. ${ }^{13}$

Following the seminal study by TK92, as well as the subsequent developments in Köbberling and Wakker (2005) and ABL, we assume that the value function $v($.$) is composed of the loss$

\footnotetext{
${ }^{9}$ For the sake of illustration, consider a value function $v(x)=x^{\alpha}$ and $v(x)=-\lambda(-x)^{\beta}$ in the gain and loss domains, respectively. $\alpha$ and $\beta$ are the curvature parameters of power utility functions and $\lambda$ is the loss aversion parameter. An initial database in which all outcomes are expressed in euros yields the estimated parameters $\left(\alpha_{1}, \beta_{1}, \lambda_{1}\right)$. Now, suppose that we express the same data in another currency (e.g., dollars) by multiplying all outcomes in the database by an exchange rate $\mu>0$. Estimating parameters using this transformed dataset now yields $\left(\alpha_{1}, \beta_{1}, \lambda_{1} \times \mu^{\alpha_{1}-\beta_{1}}\right)$. Hence, the estimated value of loss aversion depends on the unit (e.g., currency) in which the outcomes is expressed unless partial reflection (i.e., $\alpha=\beta$ ) holds.

${ }^{10}$ This issue also applies to the parametric methods in TK92 and Fehr-Duda et al. (2006) which estimate the utility function in the gain and loss domains in two separate steps. Other parametric methods do not suffer from this problem (Harrison and Rutström, 2008; Post et al., 2008; Tanaka et al., 2010).

${ }^{11} \mathrm{As}$ a result of this one-step procedure, our method also allows the researcher to test whether the probability weighting functions are the same in the gain and loss domains. It also allows for testing the duality of the probability weighting function under RDU.

${ }^{12}$ This notation is related to decision under risk. In the case of decisions under uncertainty, one would simply replace $p$ and $1-p$ by $E$ and $E^{c}$ respectively. $E$ denotes an event in a state space $\Omega$ and $E^{c}$ denotes its complement in $\Omega$. In that case, $L=\left(x, y ; E, E^{c}\right)$ is a binary prospect that gives outcome $x$ if $E$ occurs, and $y$ otherwise.

${ }^{13} \mathrm{CPT}$ makes no explicit link between weighting functions $w^{+}($.$) and w^{-}($.$) which makes it more general than$ OPT in which $w^{+}(p)=w^{-}(p)$, or RDU that includes the duality condition $w^{+}(p)=1-w^{-}(1-p)$.
} 
Table 1: Summary of literature review on semi-parametric and non-parametric methods

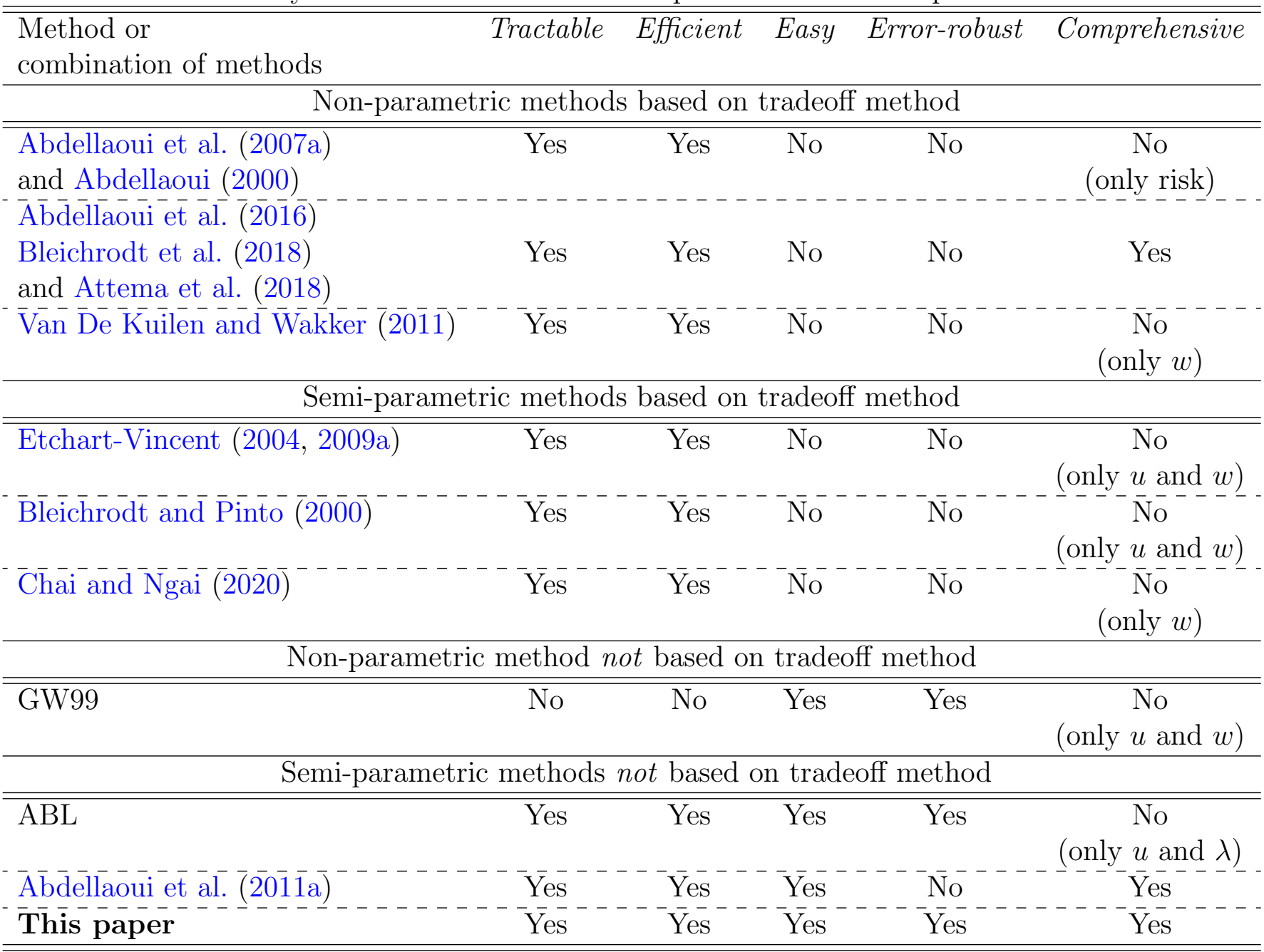


aversion index $\lambda>0$ which reflects the exchange rate between gain and loss utility units, and the utility function $u($.$) that reflects the intrinsic value of outcomes:$

$$
v(x)= \begin{cases}u(x) & \text { if } x \geq 0 \\ \lambda u(x) & \text { if } x<0\end{cases}
$$

\subsection{Elicitation}

We start by considering two standard utility functions that have been previously shown to provide a good fit to experimental data: the power utility function (see, e.g., GW99; Stott, 2006) and the exponential utility function (see, e.g., Attema et al., 2013). ${ }^{14}$ Next, we detail the steps for estimating the probability weighting function, the curvature of the utility function, and the loss aversion index.

\subsubsection{Utility function}

As in Booij et al. (2010), we use the following notation for the power (4) and exponential (5) utility functions:

$$
\begin{gathered}
u(x)=\left(\mathbf{1}_{(x \geq 0)}-\mathbf{1}_{(x<0)}\right)|x|^{\alpha_{p} \mathbf{1}_{(x \geq 0)}+\beta_{p} \mathbf{1}_{(x<0)}} \\
u(x)=\left(\mathbf{1}_{(x \geq 0)}-\mathbf{1}_{(x<0)}\right) \frac{1-\exp \left(\left(\beta_{e} \mathbf{1}_{(x<0)}-\alpha_{e} \mathbf{1}_{(x \geq 0)}\right) x\right)}{\alpha_{e} \mathbf{1}_{(x \geq 0)}+\beta_{e} \mathbf{1}_{(x<0)}}
\end{gathered}
$$

where $\mathbf{1}_{(.)}$refers to the indicator function. The important properties of these functions are related to domain-specific curvature, loss aversion, and partial reflection, and can be summarized as follows. For gains (losses), the power function in (4) is concave if $\alpha_{p}<1\left(\beta_{p}>1\right)$, linear if $\alpha_{p}=1\left(\beta_{p}=1\right)$, and convex if $\alpha_{p}>1\left(\beta_{p}<1\right)$. For gains (losses), the exponential function in (5) is concave if $\alpha_{e}>0\left(\beta_{e}<0\right)$, linear if $\alpha_{e} \longrightarrow 0\left(\beta_{e} \longrightarrow 0\right)$, and convex if $\alpha_{e}<0$ $\left(\beta_{e}>0\right)$. Furthermore, the two functions imply two different definitions of loss aversion. For (4), the loss aversion index is $\lambda=-\frac{v(-\$ 1)}{v(\$ 1)}$ which corresponds to the standard definition in TK92. For (5), the loss aversion index is given by the definition in Köbberling and Wakker (2005), that is $\lambda=\frac{v_{\uparrow}^{\prime}(0)}{v_{\downarrow}^{\prime}(0)}$ with $v_{\uparrow}^{\prime}(0)$ and $v_{\downarrow}^{\prime}(0)$ representing the left and right derivatives of the value function at the reference point. ${ }^{15}$ Finally, partial reflection corresponds to $\alpha_{p}=\beta_{p}$ and $\alpha_{e}=\beta_{e}$ in (4) and (5).

\subsubsection{Estimating probability weighting functions and utility curvature}

The first step of our estimation procedure consists of three parts. First, we select the set of probabilities $\left\{p_{k}: k=1,2, \ldots, K\right\}$ for which weights are estimated in the gain and loss domains, with $p_{k}<p_{k+1}$. For any $p_{k}$, its complement $1-p_{k}$ must also be included in the set of probabilities, so that $p_{K-k+1}=1-p_{k}$ for $k=1,2, \ldots, K$. Then, in a given domain one elicits (at least) two certainty equivalents for each probability $p_{k}$ :

$$
c e_{j, k}^{i} \sim\left(x_{j, k}^{i}, y_{j, k}^{i} ; p_{k}, 1-p_{k}\right), j=1,2, \ldots, N_{k}^{i} \quad \text { and } \quad N_{k}^{i} \geq 2
$$

\footnotetext{
${ }^{14}$ Note, however, that our method is compatible with any utility function.

${ }^{15}$ Regardless of the exact definition, it is always the case that loss aversion (loss seeking) corresponds to $\lambda>1(\lambda<1)$, whereas $\lambda=1$ captures loss neutrality.
} 
where $N_{k}^{i}$ stands for the number of certainty equivalents for $p_{k}$ in domain $i \in\{$ "+";"_" $\}$, and $x_{j, k}^{i}$ and $y_{j, k}^{i}$ are outcomes such that $x_{j, k}^{+}>y_{j, k}^{+} \geq 0$ and $x_{j, k}^{-}<y_{j, k}^{-} \leq 0 .{ }^{16}$

Thus, in total one needs to elicit $N^{+}=\sum_{k=1}^{K} N_{k}^{+} \geq 2 \times K$ certainty equivalents in the gain domain and $N^{-}=\sum_{k=1}^{K} N_{k}^{-} \geq 2 \times K$ certainty equivalents in the loss domain. For invertible $u$ and using (1) and (3), these certainty equivalents satisfy the following condition:

$$
c e_{j, k}^{i}=u^{-1}\left[\left(u\left(x_{j, k}^{i}\right)-u\left(y_{j, k}^{i}\right)\right) w^{i}\left(p_{k}\right)+u\left(y_{j, k}^{i}\right)\right]
$$

Let $\boldsymbol{c e}, \boldsymbol{x}$ and $\boldsymbol{y}$ be column vectors containing all the realizations of $c e_{j, k}^{i}, x_{j, k}^{i}$ and $y_{j, k}^{i}$, respectively. Any column vector $\mathbf{z} \in\{\boldsymbol{c e}, \boldsymbol{x}, \boldsymbol{y}\}$ is constructed as follows:

$$
\boldsymbol{z}=\left(\begin{array}{c}
\boldsymbol{z}_{1}^{+} \\
\boldsymbol{z}_{2}^{+} \\
\cdot \\
\cdot \\
\cdot \\
\boldsymbol{z}_{K}^{+} \\
\boldsymbol{z}_{1}^{-} \\
\boldsymbol{z}_{2}^{-} \\
\cdot \\
\cdot \\
\cdot \\
\boldsymbol{z}_{K}^{-}
\end{array}\right) \quad \text { with } \boldsymbol{z}_{k}^{+}=\left(\begin{array}{c}
z_{1, k}^{i} \\
z_{2, k}^{i} \\
\cdot \\
\cdot \\
\cdot \\
z_{N_{k}^{i}, k}^{i}
\end{array}\right), i \in\{"+\cdots ;-"\} \text { and } k=1,2, \ldots, K
$$

As in Hey et al. (2009) and ABL, we assume that certainty equivalents are observed with additive and normally distributed response error e. Thus, the empirical counterpart of (7) is given by:

$$
\boldsymbol{c e}_{l}=u^{-1}\left[\left(u\left(\boldsymbol{x}_{l}\right)-u\left(\boldsymbol{y}_{l}\right)\right) \times\left(\sum_{k=1}^{K}\left(\delta_{k}^{+} \boldsymbol{D}_{l}^{+}+\delta_{k}^{-} \boldsymbol{D}_{l}^{-}\right) \boldsymbol{I}_{l}^{k}\right)+u\left(\boldsymbol{y}_{l}\right)\right]+\boldsymbol{e}_{l}
$$

where $\boldsymbol{I}^{k}$ is a dummy variable set to 1 if the probability equals $p_{k}$ and 0 otherwise, $\boldsymbol{D}^{+}\left(\boldsymbol{D}^{-}\right)$ is a dummy variable set to 1 for positive (negative) certainty equivalent and 0 otherwise, and $\boldsymbol{l}$ is the $l^{\text {th }}$ line in $\boldsymbol{c e}, \boldsymbol{x}, \boldsymbol{y}$ and $\boldsymbol{I}^{k}$. Probability weights correspond to: ${ }^{17}$

$$
w^{+}\left(p_{k}\right)=\delta_{k}^{+} \text {and } w^{-}\left(p_{k}\right)=\delta_{k}^{-} \text {for } k=1, . ., K
$$

Note that $u^{-1}$ in (8) should be written in the full domain. To do that, one can first write $u$ in the full domain using indicator functions as in (4) and (5), and $u^{-1}$ can be derived using standard algebra. Finally, the parameters in (8) can be estimated as long as one provides a functional form for $u$ (and thus for $u^{-1}$ ), such as (4) or (5). ${ }^{18}$

\footnotetext{
${ }^{16}$ Note that having the same outcomes for each probability $\left(x_{j, k}^{i}=x_{j}^{i}\right.$ and $y_{j, k}^{i}=y_{j}^{i}$ for all $k$ ) allows for an immediate test of the monotonicity of preferences by checking if certainty equivalents increase with probabilities for given pairs of outcomes $\left(x_{j}^{i}, y_{j}^{i}\right)$. This choice of outcomes could also reduce the cognitive burden of the task.

${ }^{17}$ Take a probability $p_{s}$ for $s \in 1,2, \ldots, K . \boldsymbol{I}_{l}^{s}$ equals 1 for any observation $l$ that involves $p_{s}$, and all the other probability dummy variables $\boldsymbol{I}_{l}^{c}$ for $c \neq s$ are set to 0 . In that case, we have $\sum_{k=1}^{K}\left(\delta_{k}^{+} \boldsymbol{D}_{l}^{+}+\delta_{k}^{-} \boldsymbol{D}_{l}^{-}\right) \boldsymbol{I}_{j}^{k}=$ $\delta_{s}^{+} \boldsymbol{D}_{l}^{+}+\delta_{s}^{-} \boldsymbol{D}_{l}^{-}$. In the gain domain, the dummy variable $\boldsymbol{D}_{l}^{+}$equals 1 while $\boldsymbol{D}_{l}^{-}$equals 0 so that we get $\sum_{k=1}^{K}\left(\delta_{k}^{+} \boldsymbol{D}_{l}^{+}+\delta_{k}^{-} \boldsymbol{D}_{l}^{-}\right) \boldsymbol{I}_{j}^{k}=\delta_{s}^{+} \boldsymbol{D}_{l}^{+}+\delta_{s}^{-} \boldsymbol{D}_{l}^{-}=\delta_{s}^{+}$. Hence, $w^{+}\left(p_{k}\right)=\delta_{k}^{+}$in $(8)$ for $k=1,2, \ldots, K$. Analogously, $w^{-}\left(p_{k}\right)=\delta_{k}^{-}$in $(8)$ for $k=1,2, \ldots, K$.

${ }^{18}$ One can choose the functional form that best performs in terms of goodness of fit and information criteria (e.g. Hey and Orme, 1994; Fehr-Duda et al., 2006; Stott, 2006).
} 
To estimate equation 8 , we use the nonlinear least squares (NLS) method which is standard in this literature (e.g. TK92; ABL; GW99; Attema et al., 2013, 2016). ${ }^{19}$

Inserting these elements into (8), one obtains the following certainty equivalent equations for power (10) and exponential (11) utility functions:

$$
\begin{aligned}
& c e_{l}=\left(D_{l}^{+}-D_{l}^{-}\right)\left[\left(\left|\boldsymbol{x}_{l}\right|^{\alpha_{p} D_{l}^{+}+\beta_{p} D_{l}^{-}}-\left|\boldsymbol{y}_{l}\right|^{\alpha_{p} D_{l}^{+}+\beta_{p} D_{l}^{-}}\right)\left(\sum_{k=1}^{K}\left(\delta_{k}^{+} \boldsymbol{D}_{l}^{+}+\delta_{k}^{-} \boldsymbol{D}_{l}^{-}\right) \boldsymbol{I}_{l}^{k}\right)+\left|\boldsymbol{y}_{l}\right|^{\alpha_{p} D_{l}^{+}+\beta_{p} D_{l}^{-}}\right]^{\frac{1}{\alpha_{p} D_{l}^{+}+\beta_{p} D_{l}^{-}}}+\mathbf{e}_{l} \\
& c \boldsymbol{e}_{l}=\frac{\ln \left[\left(\exp \left(\left(\beta_{e} \boldsymbol{D}_{l}^{-}-\alpha_{e} \boldsymbol{D}_{l}^{+}\right) \boldsymbol{x}_{l}\right)-\exp \left(\left(\beta_{e} \boldsymbol{D}_{l}^{-}-\alpha_{e} \boldsymbol{D}_{l}^{+}\right) \boldsymbol{y}_{l}\right)\right)\left(\sum_{k=1}^{K}\left(\delta_{k}^{+} \boldsymbol{D}_{l}^{+}+\delta_{k}^{-} \boldsymbol{D}_{l}^{-}\right) \boldsymbol{I}_{l}^{k}\right)+\exp \left(\left(\beta_{e} \boldsymbol{D}_{l}^{-}-\alpha_{e} \boldsymbol{D}_{l}^{+}\right) \boldsymbol{y}_{l}\right)\right]}{\beta_{e} \boldsymbol{D}_{l}^{-}-\alpha_{e} \boldsymbol{D}_{l}^{+}}+\mathbf{e}_{l}
\end{aligned}
$$

\subsubsection{Estimating loss aversion}

As a second step, we measure the loss aversion index $\lambda$ as defined in (3) based on the estimates of the utility function and the probability weights as outlined in Section 3.2.2. Following Abdellaoui et al. (2007a), the estimation of the loss aversion index can be done using a set of $K$ indifference relationships that involve mixed binary prospects:

$$
c e_{k}^{*} \sim\left(x_{k}^{*}, y_{k}^{*} ; p_{k}, 1-p_{k}\right), \quad k=1,2, \ldots, K
$$

with $y_{k}^{*}<0<x_{k}^{*}$. Under CPT, these indifferences imply that:

$$
c e_{k}^{*}=v^{-1}\left[w^{+}\left(p_{k}\right) v\left(x_{k}^{*}\right)+w^{-}\left(1-p_{k}\right) v\left(y_{k}^{*}\right)\right]
$$

Because $c e_{k}^{*}$ belongs to the interval $\left(y_{k}^{*}, x_{k}^{*}\right)$, it could either be a gain or a loss. Also, note that for each $k$ both $w^{+}\left(p_{k}\right)$ and $w^{-}\left(1-p_{k}\right)$ are known since they have been elicited in the previous step. Echoing our previous notation, let $\boldsymbol{c} \boldsymbol{e}^{*}, \boldsymbol{x}^{*}$ and $\boldsymbol{y}^{*}$ contain the realizations of $c e_{k}^{*}, x_{k}^{*}$ and $y_{k}^{*}$. In addition, denote by $\boldsymbol{\delta}^{+}$and $\boldsymbol{\delta}^{-}$the column vectors such that $\boldsymbol{\delta}^{+^{\prime}} \equiv\left(\delta_{1}^{+}, \delta_{2}^{+}, \ldots, \delta_{K}^{+}\right)$and $\underline{\boldsymbol{\delta}}^{{ }^{\prime}} \equiv\left(\underline{\delta}_{1}^{-}, \underline{\delta}_{2}^{-}, \ldots, \underline{\delta}_{K}^{-}\right)=\left(\delta_{K}^{-}, \delta_{K-1}^{-}, \ldots, \delta_{1}^{-}\right) .{ }^{20}$ Assuming that certainty equivalents are observed with an additive and normally distributed response error term $\left(\boldsymbol{e}_{k}\right)$, the empirical counterpart of equation (13) then becomes:

$$
\boldsymbol{c e}_{k}^{*}=v^{-1}\left[\boldsymbol{\delta}_{k}^{+} v\left(\boldsymbol{x}_{k}^{*}\right)+\underline{\boldsymbol{\delta}}_{k}^{-} v\left(\boldsymbol{y}_{k}^{*}\right)\right]+\boldsymbol{e}_{k}
$$

$v^{-1}$ in (14) can be derived similarly to $u^{-1}$ in (8). Then, the respective certainty equivalent equations for power and exponential utility functions become:

$$
\begin{gathered}
\boldsymbol{c} \boldsymbol{e}_{k}^{*}=\left(\boldsymbol{D}_{k}^{+}-\boldsymbol{D}_{k}^{-}\right)\left[\frac{\boldsymbol{\delta}_{k}^{+}\left(\boldsymbol{x}_{k}^{*}\right)^{\alpha_{p}}-\lambda \underline{\boldsymbol{\delta}}_{k}^{-}\left(-\boldsymbol{y}_{k}^{*}\right)^{\beta_{p}}}{\boldsymbol{D}_{k}^{+}-\lambda \boldsymbol{D}_{k}^{+}}\right]^{\frac{1}{\alpha_{p} \boldsymbol{D}_{k}^{+}+\beta_{p} \boldsymbol{D}_{k}^{-}}}+\boldsymbol{e}_{k} \\
\boldsymbol{c} \boldsymbol{e}_{k}^{*}=\frac{\ln \left[1-\frac{\alpha_{e} \boldsymbol{D}_{k}^{+}+\beta_{e} \boldsymbol{D}_{k}^{-}}{\boldsymbol{D}_{k}^{+}-\lambda \boldsymbol{D}_{k}^{-}}\left(\boldsymbol{\delta}_{k}^{+}\left(\frac{1-\exp \left(-\alpha_{e} \boldsymbol{x}_{k}^{*}\right)}{\alpha_{e}}\right)-\lambda \underline{\boldsymbol{\delta}}_{k}^{-}\left(\frac{1-\exp \left(\beta_{e} \boldsymbol{y}_{k}^{*}\right)}{\beta_{e}}\right)\right)\right]}{\beta_{e} \boldsymbol{D}_{k}^{-}-\alpha_{e} \boldsymbol{D}_{k}^{+}}+\boldsymbol{e}_{k}
\end{gathered}
$$

Using the values of the probability weights and the parameters of $u($.$) from the first step, we$ can estimate (15) or (16) by NLS to obtain $\lambda$.

\footnotetext{
${ }^{19}$ We also note that some studies used maximum likelihood estimator (see Fehr-Duda et al., 2006; Hey et al., 2009). Although the latter method has appealing asymptotic properties (including efficiency), it requires more stringent distributional assumptions as well as large samples. Under the usual assumption of normally distributed response errors, MLE and NLS will yield the same point estimates of risk attitudes in equation (8) since they both rely of the same first order conditions, and MLE yields lower standard errors than NLS (in line with the aforementioned efficiency argument). Importantly, all the statistical results reported in section 4 that are based on the NLS procedure also hold under MLE.

${ }^{20}$ We used underscore just to change subscript in loss domain : $\underline{\delta}_{k}^{-} \equiv \delta_{K-k+1}^{-}=w^{-}\left(1-p_{k}\right)$ for $k=1,2, \ldots, K$.
} 


\subsubsection{Key properties of our method}

Comparison with ABL. We refine and extend the method previously proposed by ABL in several ways.

First, unlike ABL, we can estimate multiple probability weights and thus elicit the shape of the probability weighting function.

Second, our method uses a single step to estimate the probability weights and the utility function in the full domain, whereas ABL propose a two-step procedure. This feature of our method allows for testing several important restrictions (partial reflection, identical probability weighting functions across domains, and duality) as well as imposing these restrictions whenever necessary.

Imposing partial reflection helps avoid the problem of arbitrary measurement of loss aversion with power utility functions, as raised by Wakker (2010). Testing for identical probability weighting functions across domains (i.e., $w^{+}\left(p_{k}\right)=w^{-}\left(p_{k}\right)$ for all $k$ ) allows us to test for a key assumption of OPT. In addition, this assumption must also be made under CPT whenever loss aversion is present and preferences are homogenous (Al-Nowaihi et al., 2008). ${ }^{21}$ Our method allows for testing and imposing duality $\left(\right.$ i.e., $w^{+}\left(p_{k}\right)=1-w^{-}\left(1-p_{k}\right)$ for all $\left.k\right){ }^{22}$ By allowing for testing and imposing duality as well as identical probability weighting across domains, our method can be applied under RDU (Quiggin, 1982; Gilboa, 1987; Schmeidler, 1989) and OPT (KT79). This is not possible using existing parametric, semi-parametric, or non-parametric methods.

Third, certainty equivalents for mixed prospects are obtained using a different procedure than the one proposed by ABL. In ABL, subjects are asked to provide a loss amount $L^{*}$ for which they are indifferent between the status-quo $(0)$ and a binary lottery $\left(G^{*}, L^{*} ; p_{g}, 1-p_{g}\right)$ where $G^{*}$ is a fixed gain and $L^{*} \in(-\infty, 0]$ is a loss. In this elicitation procedure, the researcher does not know the lower bound of the loss interval. By contrast, equation (12) keeps track of the upper and lower bounds of the loss interval because $c e_{k}^{*}$ belongs to $\left(y_{k}^{*}, x_{k}^{*}\right)$. This is an appealing property of our method for two reasons. First, asking subjects to provide indifference values on unbounded intervals can be cognitively demanding (Abdellaoui et al., 2007a; Wakker and Deneffe, 1996). This may lead to errors that potentially inflate the estimates of loss aversion, as reported by ABL (see Table 11, pp. 263-264). Second, eliciting indifference values on bounded intervals allows us to use standard switching outcome procedure (Booij and Van de Kuilen, 2009).

Comparison to standard parametric methods. Our method retains all the appealing properties of parametric methods. First, it is as efficient as parametric methods because, for $\mathrm{K}$ probability weights to be elicited, the smallest number of certainty equivalents required to measure all the three components of risk attitudes in the full domain is $5 K$ (i.e., $2 K$ certainty equivalents in the gain domain, $2 K$ in the loss domain and $K$ for mixed lotteries). Second, we use simple questions (comparisons of certain outcomes and binary lotteries) so that the method is not cognitively demanding for subjects. Third, our method is tractable because we can measure risk attitudes using standard regression techniques (i.e., nonlinear least squares). Fourth, our estimation method accounts for response errors.

In parametric methods, it is key to assess the validity of the functional forms used for the probability weighting and utility functions. However, parametric methods do not allow the researcher to separately assess the goodness of fit of each of these functions (GW99). In contrast, our semi-parametric method does not make any parametric assumption regarding

\footnotetext{
${ }^{21}$ Homogeneity of preferences holds whenever multiplying all the payoffs of a non-mixed lottery by a positive constant $c$ also leads the certainty equivalent of the lottery to be multiplied by $c$.

${ }^{22}$ As pointed out by Abdellaoui (2000, pp. 1509-1510), testing for duality with parametric methods and nonparametric methods based on the tradeoff approach requires using the specific probability weighting function of Goldstein and Einhorn (1987) and Lattimore et al. (1992).
} 
the probability weighting function and allows the researcher to evaluate the goodness of fit of the utility function separately. Thus, one can select the utility function with the best fit, further improving the accuracy of the elicitation of risk attitudes of the semi-parametric method compared to the non-parametric one.

Applicability to unknown probabilities. Our method is also directly applicable to cases of uncertainty where probabilities are unknown. It does not require setting any specific conditions on the event space, and hence can be applied to real-life uncertainty situations (Baillon et al., 2018). ${ }^{23}$ Extending our method to the case of uncertainty can thus be done by simply replacing probability dummy variables by event dummy variables in equations (8) and (14).

\section{Application}

We apply our method to the data of TK92 who collected 64 indifference values from each of their 25 subjects. TK92 provided parametric estimates for each subject and then reported median values across subjects. The aim of this application is to compare the elicitation results of risk attitudes using the parametric method of TK92 with our method. We use 62 of the 64 median indifference values in TK92 to the two methods. ${ }^{24}$

\subsection{Curvature of the utility function for gains and losses}

In Table 2, we report our parametric and semi-parametric estimations of the CPT components of the value function (curvature in each domain and loss aversion) for two parametric specifications of the utility function (power and exponential). ${ }^{25}$ All statistical tests in the paper are twosided. ${ }^{26}$

Power utility function. Our semi-parametric estimations suggest that the utility function is concave in the gain domain since the point estimate of $\alpha_{p}=0.904$ is significantly lower than one $(p-$ value $=0.043)$. In the loss domain, the estimated value of $\beta_{p}=1.069$ points to the concavity of the utility function, which is in line with the findings of ABL, Attema et al. (2013) and Etchart-Vincent and l'Haridon (2011). However, in statistical terms we cannot reject the null hypothesis that $\beta_{p}$ equals one, pointing to the linearity of the utility function in the loss domain $(p-$ value $=0.262)$. By contrast, parametric estimations imply a concave utility function in the gain domain $\left(\alpha_{p}=0.839, p-\right.$ value $\left.<0.001\right)$ and a convex function in the loss domain $\left(\beta_{p}=0.906, p-\right.$ value $\left.=0.003\right)$. Furthermore, based on our semi-parametric method, we reject the null hypothesis of partial reflection $\left(\alpha_{p}=\beta_{p}, p\right.$-value $\left.=0.036\right)$ whereas this is not the case for the parametric method $(p-$ value $=0.110)$. To avoid arbitrary measurements of loss aversion under power utility function, partial reflection should, however, be imposed (e.g. Harrison and Rutström, 2008; Post et al., 2008; Tanaka et al., 2010). When imposing partial reflection, our semi-parametric method yields $\alpha_{p}=\beta_{p}=0.976$. In purely descriptive terms, this is consistent with the standard finding of the S-shaped value function (concavity in the gain domain and convexity in the loss domain). However, we cannot reject the linearity hypothesis

\footnotetext{
${ }^{23}$ Previous methods require that the universal event is an interval of real numbers (e.g. temperature in a town) which is most suitable to deal with artificial uncertainty situations that can be created in the laboratory (Van De Kuilen and Wakker, 2011).

${ }^{24}$ The two excluded indifference values correspond to problems 7 and 8 in Table 6 of TK92. These indifference values are not compatible with our method since they involve comparing two binary lotteries in the gain domain, while equation (8) requires information about the certainty equivalent.

${ }^{25}$ Detailed results for the semi-parametric method are reported in Appendix A1.2. In addition, Appendix A1.4 outlines the estimation procedure and the results for the parametric method.

${ }^{26}$ We use t-test to test whether a coefficient is equal to a specific value and whether the difference between two coefficients is significant. We use F-test to test joint hypotheses on coefficient values.
} 
$\left(\alpha_{p}=\beta_{p}=1, p\right.$-value $\left.=0.531\right)$. With the parametric method, imposing partial reflection leads to $\alpha_{p}=\beta_{p}=0.872$ which is significantly different from $1(p-$ value $<0.001)$. Interestingly, this parametric measurement on median data of utility curvature closely corresponds to the median result on individual data (0.88) reported by TK92. ${ }^{27}$

Exponential utility function. Our semi-parametric method leads to the following estimates in the gain and loss domains: $\alpha_{e}=0.00158$ and $\beta_{e}=-0.00154$. These values suggest concavity of utility in both the gain and loss domains. However, we cannot reject the null hypothesis of linearity in both the gain domain $\left(\alpha_{e}=0, p-\right.$ value $\left.=0.161\right)$ and in the loss domain $\left(\beta_{e}=0, p-\right.$ value $\left.=0.187\right)$. The joint hypothesis of linearity of utility in full domain $\left(\alpha_{e}=\beta_{e}=0\right)$ cannot be rejected $(p-$ value $=0.1604)$. In contrast, the parametric method implies concavity in the gain domain $\left(\alpha_{e}=0.00168, p-\right.$ value $\left.=0.013\right)$ and convexity in the loss domain $\left(\beta_{e}=0.00155, p-\right.$ value $\left.=0.030\right)$. Partial reflection $\left(\alpha_{e}=\beta_{e}\right)$ cannot be rejected $(p-$ value $=0.898)$.

Table 2: Summary of estimation results of the utility function and of loss aversion

\begin{tabular}{|c|c|c|c|}
\hline \multirow{2}{*}{\multicolumn{4}{|c|}{\begin{tabular}{cc}
\multicolumn{2}{c}{ Power utility } \\
No constrain $\quad$ Constrain $\alpha_{p}$
\end{tabular}}} \\
\hline & & & \\
\hline \multicolumn{4}{|c|}{ Our semi-parametric measurements on median data ${ }^{\dagger}$} \\
\hline Utility parameter (Gain) & 0.904 & 0.976 & 0.00158 \\
\hline Utility parameter (Loss) & 1.069 & 0.976 & -0.00154 \\
\hline Loss aversion $\lambda$ & 0.884 & 1.751 & 1.679 \\
\hline AIC criterion ${ }^{\star}$ & 281 & 287 & 283 \\
\hline \multicolumn{4}{|c|}{ Parametric measurements on median data } \\
\hline Utility parameter (Gain) & 0.839 & 0.872 & 0.00168 \\
\hline Utility parameter (Loss) & 0.906 & 0.872 & 0.00155 \\
\hline Loss aversion $\lambda$ & 1.256 & 1.730 & 1.774 \\
\hline AIC criterion ${ }^{\star}$ & 318 & 318 & 337 \\
\hline \multicolumn{4}{|c|}{ Median individual estimates reported by TK92 } \\
\hline Utility parameter (Gain) & 0.88 & & \\
\hline Utility parameter (Loss) & 0.88 & & \\
\hline Loss aversion $\lambda$ & 2.25 & & \\
\hline
\end{tabular}

$\dagger$ TK92 collected 64 indifference values from each of the 25 subjects they have in the experiment. For each of the 64 indifference values, they reported the median value. We used 62 median values in TK92 to perform our semi-parametric method and parametric method.

$\ddagger$ TK92 estimated $\alpha_{p}, \beta_{p}, \lambda$ and the parameters of the probability weighting function for each subject and then reported the median value for each estimate.

* AIC stands for Akaike Information Criterion

\subsection{Loss aversion}

\subsubsection{Our semi-parametric method}

Under the power utility function and without imposing partial reflection, our semi-parametric method estimates the loss aversion index to be $\lambda=0.884$. This would mean that the median subject exhibits loss seeking instead of loss aversion. However, this estimated loss aversion is subject to the problem of arbitrary measurement (Wakker, 2010; Köbberling and Wakker, 2005). We circumvent this issue by imposing partial reflection (e.g. Harrison and Rutström,

\footnotetext{
${ }^{27}$ TK92 do not impose partial reflection for each of the 25 subjects, but they found that the median estimates of utility curvature are the same across domains (e.g. Wakker, 2010, pp. 270)).
} 
2008; Post et al., 2008; Tanaka et al., 2010) and find $\lambda=1.751$ which points to the presence of loss aversion. In the same vein, for the exponential utility specification we find $\lambda=1.679$.

\subsubsection{Parametric method of TK92}

Using the power utility function without imposing partial reflection, the loss aversion index is $\lambda=1.256$, suggesting (mild) loss aversion. Imposing partial reflection yields $\lambda=1.730$ in line with the presence of loss aversion. Using the exponential utility specification, we estimate $\lambda=1.774$ which also confirms that subjects exhibit loss aversion. These parametric estimates of loss aversion are lower than the median estimate previously reported by TK92. However, their median loss aversion index of 2.25 should be taken with a grain of salt because partial reflection would not be expected to hold for all 25 individual estimates (e.g. Wakker, 2010, pp. 270). Consequently, loss aversion is likely to have been arbitrarily measured for some individuals. We thus maintain 1.73 as the point estimate of loss aversion index for the parametric method.

\subsection{Probability weighting function}

\subsubsection{Our semi-parametric method}

The AIC values reported in Table 2 suggest that the best fitting specification of the utility function is for the power utility function without the constraint of partial reflection. ${ }^{28}$ Figure 1 presents the estimates of the probability weighting function across domains under this specification (labelled Semi-para). ${ }^{29}$ We obtain overweighting for $p \in(0,0.25]$ and underweighting for $p \in(0.25,1)$ in the gain domain. Furthermore, probabilistic risk neutrality $w^{+}(p)=p$ is rejected for all probabilities (all $p$-values $<0.05$ ) except for 0.1 and 0.25 . We reject the joint hypothesis of linearity of the probability weighting function over the whole range of probabilities in the gain domain $(p-$ value $<0.001)$. In the loss domain, the linearity of the probability weighting function $\left(w^{-}(p)=p\right)$ is rejected for all probabilities $(p-$ values $<0.05)$ except for 0.1. We observe overweighting for $p \in(0,0.1)$ and underweighting for $p \in(0.1,1)$. We also reject the joint hypothesis of linearity of the probability weighting function over the whole range of probabilities in the loss domain $(p-$ value $<0.001)$.

The equality of probability weights $w^{+}\left(p_{k}\right)=w^{-}\left(p_{k}\right)$ across domains is rejected for probabilities $0.05,0.25$ and 0.90 (all $p$-values $<0.01$ ) but cannot be rejected for probabilities 0.01 , $0.1,0.5,0.75,0.95$ and 0.99 (all $p$-values $>0.079$ ). The joint hypothesis of the equality of probability weights over all probabilities is rejected $(p-$ value $<0.001)$.

Duality $w^{+}\left(p_{k}\right)=1-w^{-}\left(1-p_{k}\right)$ is rejected for all probabilities (all $p-$ values $<0.001$ ) except for 0.01 and 0.05 (all $p$-values $>0.119$ ). The joint hypothesis of duality over all probabilities is rejected $(p-$ value $<0.001)$.

We report the estimates of the probability weighting function under the constraints of identical probability weightings and duality in Appendix A1.3. Using AIC as a goodness of fit measure, we find that specification based on CPT (i.e., different probability weighting across domains) outperforms OPT (i.e, identical probability weighting) and RDU (i.e., duality assumption), while OPT outperforms RDU. This result echoes the observation of TK92 (p. 312) that both $w^{+}(0.5)$ and $w^{-}(0.5)$ are estimated to be less than 0.5 , which contradicts duality but not identical probability weighting for $p=0.5$.

\footnotetext{
${ }^{28}$ Using AIC as a goodness of fit measure is commonplace in the literature. See, inter alia, Fehr-Duda et al. (2006); Stott (2006); Hey and Orme (1994).

${ }^{29}$ See Appendix A1.2 for alternative estimates of the probability weighting function under the exponential utility function and the power utility function with partial reflection. The results remain robust under different specifications of $u$.
} 
Figure 1: Semi-parametric and parametric measurements of the probability weighting function (TK92, median data) $)^{\dagger}$
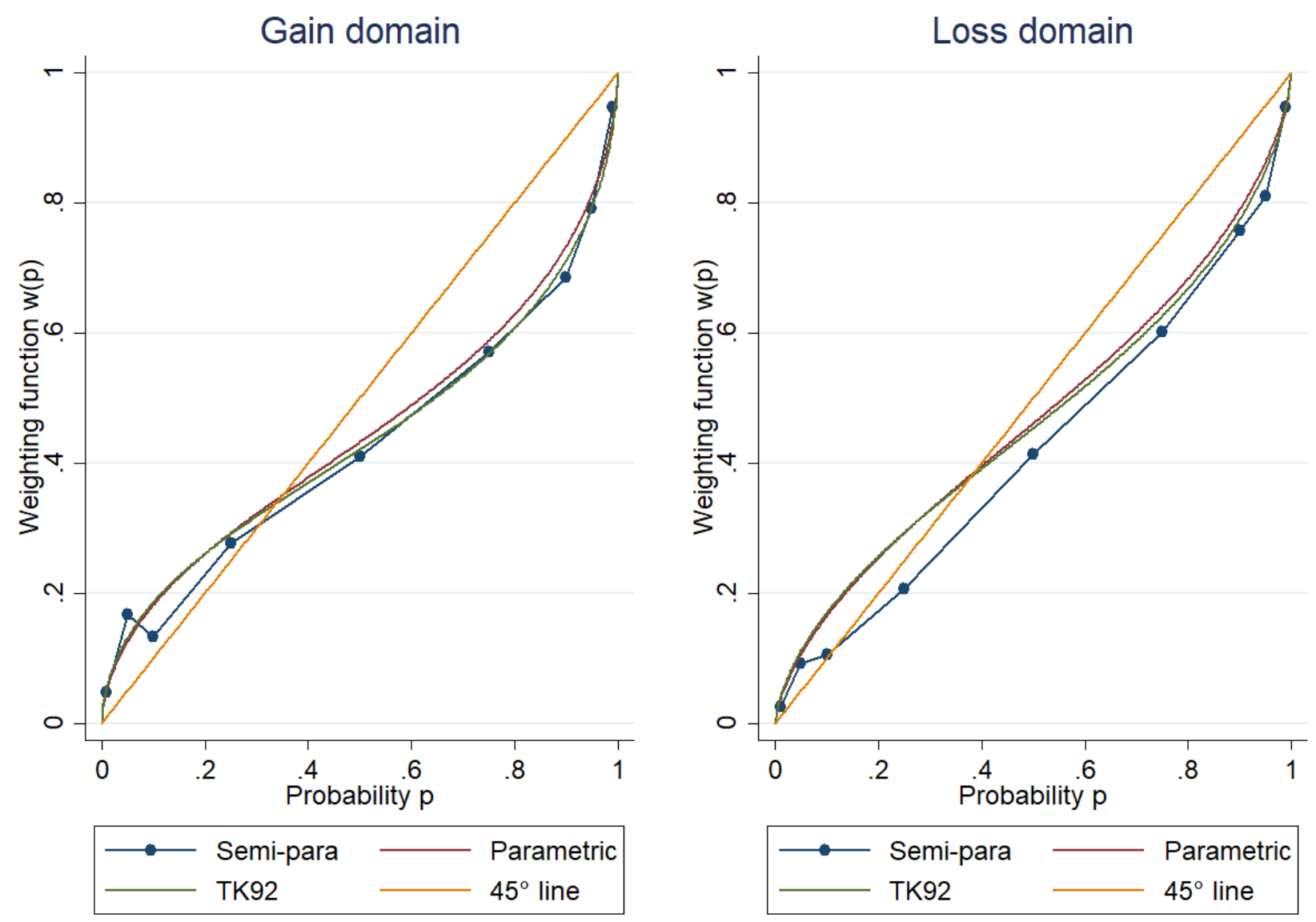

$\dagger$ Semi-para : semi-parametric method with power utility function without partial reflection constraint; Parametric: parametric method with power utility function and partial reflection constraint; TK92: parametric measurement reported by TK92

\subsubsection{Parametric method of TK92}

We now turn to discussing the remaining content presented in Figure 1: probability weighting functions of the parametric method estimated using median data (labelled Parametric), and the median results of TK92 which are based on individual data (labelled TK92). Since partial reflection cannot be rejected under parametric method (see subsection 4.1), herein we focus on the probability weighting functions based on power utility with partial reflection constraint. In Appendix A1.4, we also provide the estimates for the power utility without partial reflection constraint and for the exponential utility function. The probability weighting function used in the parametric method of TK92 is as follows:

$$
w^{i}(p)=\frac{p^{\left(\gamma \mathbf{1}_{(i=+)}+\theta \mathbf{1}_{(i=-)}\right)}}{\left[p^{\left(\gamma \mathbf{1}_{(i=+)}+\theta \mathbf{1}_{(i=-)}\right)}+(1-p)^{\left(\gamma \mathbf{1}_{(i=+)}+\theta \mathbf{1}_{(i=-)}\right)}\right]^{\frac{1}{\gamma \mathbf{1}_{(i=+)}+\theta \mathbf{1}_{(i=-)}}}}
$$

The parameters of the probability weighting function are estimated at $\gamma=0.635$ and $\theta=0.713$. These parameters are significantly different from 1 (both $p-$ values $<0.001$ ), which means that, notwithstanding the standard assumptions of EUT, subjects do not take probabilities at face value. In the gain domain, we obtain overweighting for $p \in\left(0, \frac{1}{3}\right)$, probability risk neutrality at $p=\frac{1}{3}$ and underweighting for $p \in\left(\frac{1}{3}, 1\right)$. In the loss domain, we obtain overweighting for $p \in(0,0.4)$, probability risk neutrality at $p=0.4$ and underweighting for 
$p \in(0.4,1)$. In addition, the hypothesis $\gamma=\theta$ is rejected $(p-$ value $=0.003)$, meaning that, in contrast with OPT, the probability weighting functions differ in the gain and loss domains. Our estimate of $\theta=0.713$ is not statistically different from the value $\theta=0.690$ reported by TK92 $(p-$ value $=0.1471)$. Our estimate of $\gamma=0.635$ is, however, significantly different from the value $\gamma=0.610$ reported by TK92 $(p-$ value $=0.032)$. Regarding duality, TK92 cannot provide a complete test under their one-parameter (per domain) probability weighting function (Abdellaoui, 2000, pp. 1509-1510).

On a final note, we compare our semi-parametric method estimates with the parametric method used in TK92. Looking at AIC, we find that the goodness of fit of our semi-parametric method under both the power utility $(\mathrm{AIC}=281.929$ without partial reflection constraint $)$ and the exponential utility specifications $(\mathrm{AIC}=283.986)$ substantially outperform the parametric method of TK92 (AIC $=318.997$ for power utility with partial reflection constraint and AIC $=337.134$ for exponential utility). ${ }^{30}$ Hence, we conclude that our semi-parametric method fits the data better than the parametric method.

\section{Discussion}

According to TK92, individual behavior under risk is characterized by the so-called 'fourfold pattern of risk attitudes': ( $i$ ) risk seeking (risk aversion) for small probabilities in the gain (loss) domain and ( $i i$ ) risk aversion (risk seeking) for intermediate and high probabilities in the gain (loss) domain. TK92 stress that the fourfold pattern of risk attitudes is consistent with a S-shaped utility function (i.e., concavity in the gain domain and convexity in the loss domain) and an inverse S-shaped probability weighting function. They use parametric estimates to corroborate these predictions. They also report evidence for partial reflection that has led numerous researchers to adopt this assumption in various applications (see, e.g. Corgnet and Hernan-Gonzalez, 2019; Dhami and Al-Nowaihi, 2007; Dhami, 2016, pp. 242-248). The previous section outlines the main empirical findings of our semi-parametric method and compare them with those obtained under the parametric method of TK92. Below, we discuss these findings further in light of the existing literature.

\subsection{The value function}

Our result regarding the absence of convexity of the utility function in the loss domain also contrasts with TK92. However, this finding is consistent with a number of previous studies (ABL; Bruhin et al., 2010; Abdellaoui et al., 2011a; Attema et al., 2013, 2016; Kemel and Mun, 2020). ABL, Attema et al. (2013, 2016), Abdellaoui et al. (2011a) and Kemel and Mun (2020) use the semi-parametric method developed by ABL, whereas Bruhin et al. (2010) use a parametric method with a power utility function and a two-parameter probability weighting function as in Goldstein and Einhorn (1987). In contrast, studies based on the tradeoff method have found support for the convexity of the utility function in the loss domain (Abdellaoui, 2000; Etchart-Vincent, 2004, 2009b; Abdellaoui et al., 2007a, 2013, 2016; Booij and Van de Kuilen, 2009; Hajimoladarvish, 2017; Attema et al., 2018; Bleichrodt et al., 2018). To the best of our knowledge, no studies based on the tradeoff method have yet reported concavity of the utility function in the loss domain. Hence, the convexity of the utility function found by TK92 seems not to be robust to the choice of the estimation technique. More precisely, using different probability weighting functions might explain differences in the estimates of the curvature of the utility function. In Appendix A1.5, we show that using the two-parameter probability weighting

\footnotetext{
${ }^{30}$ In other words, the semi-parametric method minimizes information loss compared to the parametric method with almost certainty.
} 
function of Goldstein and Einhorn (1987) instead of the one-parameter probability weighting function of TK92 leads to a concave utility function in the loss domain. The sensitivity of parametric results with respect to the specification of the probability weighting specification has been previously highlighted by GW99 and Abdellaoui (2000). Unlike the specification of the probability weighting function, we believe that differences regarding the specification of the utility function do not account for the absence of concavity in the utility function in the loss domain. In Appendix A2, we show that we can reproduce the non-parametric results of GW99 using our semi-parametric method, thus highlighting that the specification of the utility function does not seem to be a fundamental driver of our results. In addition, our findings hold for both the power and exponential utility functions. From a theoretical standpoint, people who exhibit a concave utility in the loss domain can still be risk seeking in the loss (Chateauneuf and Cohen, 1994). Our estimation method thus allows for such a possibility.

The empirical evidence on partial reflection in the literature is mixed. Our rejection of partial reflection is consistent with some studies (Abdellaoui et al., 2013, 2016; Attema et al., 2013, 2016, ABL) but not others (e.g. Abdellaoui, 2000; Andersen et al., 2006; Abdellaoui et al., 2007a; Harrison and Rutström, 2009; Booij and Van de Kuilen, 2009; Booij et al., 2010).

Finally, our estimate of the loss aversion index in the semi-parametric method is similar to the one elicited with the parametric method on median data $(\lambda \approx 1.7)$. This value is lower than the value of 2.25 corresponding to the median value of individual estimates in TK92. However, it is close to the estimated value of 1.6 found both by Booij et al. (2010) who use structural estimation techniques, and ABL on pooled data. It is also similar to the estimate reported by Tom et al. (2007), Pennings and Smidts (2003), Booij and Van de Kuilen (2009) and Kemel and Mun (2020): $\lambda=1.93, \lambda=1.8, \lambda=1.87$ and $\lambda=1.31$.

\subsection{The probability weighting function}

Our semi-parametric method complements the analysis in TK92 by assessing the duality condition and the equality of the probability weighting function across domains over the whole range of probabilities. We reject both duality (RDU, Quiggin, 1982; Gilboa, 1987; Schmeidler, 1989) and identical probability weighting functions across domains (OPT, Kahneman and Tversky, 1979). Tests of duality and identical probability weightings that cover the whole range of probabilities are scarce in the literature. Our findings echo Abdellaoui (2000) who reject both duality condition and identical probability weighting functions across domains under risk. However, under uncertainty, Abdellaoui et al. (2005) do not reject duality, although they do reject identical weighting functions across domains. Importantly, our rejection of both duality and identical probability weighting function provides support for CPT.

We also find a less elevated probability weighting function, regardless of the domain, as compared to TK92. More surprisingly, we also find that the probability weighting function crosses the $45^{\circ}$ line in the loss domain at $p=0.1$ which is far below the estimate of 0.4 in TK92. Our crossing point estimate of $p=0.1$ is in line with the results of Hajimoladarvish (2017, p. 51) over small loss that are obtained with tradeoff method. However, our crossing point estimate is below what is reported in other studies (e.g. Fehr-Duda et al., 2006; Abdellaoui, 2000; Bruhin et al., 2010; Booij et al., 2010).

\section{Conclusion and future research}

ABL and Abdellaoui et al. (2011b) deploy a semi-parametric method to elicit the utility function and loss aversion. In this paper, we go one step further by developing a semi-parametric method that elicits all dimensions of risk attitudes, including the whole range of probability weights. In addition, our method can be applied to both risk and uncertainty. Importantly, it retains 
the four appealing properties of the parametric method that have been discussed at length in the literature.

In the spirit of the work of Abdellaoui et al. (2011b), our semi-parametric method can also be used to extend the popular elicitation technique of Holt and Laury (2002) to the case in which probabilities are distorted. Future work will be dedicated to the formal development of this practically relevant extension.

\section{References}

Mohammed Abdellaoui. Parameter-free elicitation of utility and probability weighting functions. Management Science, 46(11):1497-1512, 2000.

Mohammed Abdellaoui, Frank Vossmann, and Martin Weber. Choice-based elicitation and decomposition of decision weights for gains and losses under uncertainty. Management Science, 51(9):1384-1399, 2005.

Mohammed Abdellaoui, Han Bleichrodt, and Corina Paraschiv. Loss aversion under prospect theory: A parameter-free measurement. Management Science, 53(10):1659-1674, 2007a.

Mohammed Abdellaoui, Han Bleichrodt, and Olivier L'Haridon. A tractable method to measure utility and loss aversion under prospect theory. 2007b.

Mohammed Abdellaoui, Han Bleichrodt, and Olivier l'Haridon. A tractable method to measure utility and loss aversion under prospect theory. Journal of Risk and Uncertainty, 36(3):245, 2008.

Mohammed Abdellaoui, Olivier L'Haridon, and Corina Paraschiv. Experienced vs. described uncertainty: Do we need two prospect theory specifications? Management Science, 57(10): 1879-1895, 2011a.

Mohammed Abdellaoui, Ahmed Driouchi, and Olivier L'Haridon. Risk aversion elicitation: reconciling tractability and bias minimization. Theory and Decision, 71(1):63-80, 2011b.

Mohammed Abdellaoui, Aurélien Baillon, Laetitia Placido, and Peter P Wakker. The rich domain of uncertainty: Source functions and their experimental implementation. American Economic Review, 101(2):695-723, 2011c.

Mohammed Abdellaoui, Han Bleichrodt, and Hilda Kammoun. Do financial professionals behave according to prospect theory? an experimental study. Theory and Decision, 74(3): 411-429, 2013.

Mohammed Abdellaoui, Han Bleichrodt, Olivier l'Haridon, and Dennie Van Dolder. Measuring loss aversion under ambiguity: A method to make prospect theory completely observable. Journal of Risk and Uncertainty, 52(1):1-20, 2016.

Mohammed Abdellaoui, Han Bleichrodt, Emmanuel Kemel, and Olivier l'Haridon. Measuring beliefs under ambiguity. Operations Research, forthcoming, 2020.

Ali Al-Nowaihi, Ian Bradley, and Sanjit Dhami. A note on the utility function under prospect theory. Economics Letters, 99(2):337-339, 2008. 
Steffen Andersen, Glenn W Harrison, and Elisabet E Rutström. Choice behavior, asset integration and natural reference points. Technical report, Working Paper 06, 2006.

Arthur E Attema, Werner BF Brouwer, and Olivier l'Haridon. Prospect theory in the health domain: A quantitative assessment. Journal of Health Economics, 32(6):1057-1065, 2013.

Arthur E Attema, Werner BF Brouwer, Olivier l'Haridon, and Jose Luis Pinto. An elicitation of utility for quality of life under prospect theory. Journal of Health Economics, 48:121-134, 2016.

Arthur E Attema, Han Bleichrodt, and Olivier L'Haridon. Ambiguity preferences for health. Health Economics, 27(11):1699-1716, 2018.

Aurélien Baillon, Zhenxing Huang, Asli Selim, and Peter P Wakker. Measuring ambiguity attitudes for all (natural) events. Econometrica, 86(5):1839-1858, 2018.

Nicolò Bertani, Abdellah Boukhatem, Enrico Diecidue, Patrice Perny, and Paolo Viappiani. Fast and simple adaptive elicitations: Experimental test for probability weighting. Available at SSRN 3569625, 2019.

Han Bleichrodt and Jose Luis Pinto. A parameter-free elicitation of the probability weighting function in medical decision analysis. Management Science, 46(11):1485-1496, 2000.

Han Bleichrodt, Olivier L'Haridon, and David Van Ass. The risk attitudes of professional athletes: Optimism and success are related. Decision, 5(2):95, 2018.

Adam S Booij and Gijs Van de Kuilen. A parameter-free analysis of the utility of money for the general population under prospect theory. Journal of Economic Psychology, 30(4):651-666, 2009.

Adam S Booij, Bernard MS Van Praag, and Gijs Van De Kuilen. A parametric analysis of prospect theory's functionals for the general population. Theory and Decision, 68(1-2):115$148,2010$.

Raphael Bostic, Richard J Herrnstein, and R Duncan Luce. The effect on the preference-reversal phenomenon of using choice indifferences. Journal of Economic Behavior \& Organization, 13(2):193-212, 1990.

Adrian Bruhin, Helga Fehr-Duda, and Thomas Epper. Risk and rationality: Uncovering heterogeneity in probability distortion. Econometrica, 78(4):1375-1412, 2010.

Junyi Chai and Eric WT Ngai. The variable precision method for elicitation of probability weighting functions. Decision Support Systems, 128:113166, 2020.

Alain Chateauneuf and Michèle Cohen. Risk seeking with diminishing marginal utility in a non-expected utility model. Journal of Risk and Uncertainty, 9(1):77-91, 1994.

Brice Corgnet and Roberto Hernan-Gonzalez. Revisiting the trade-off between risk and incentives: The shocking effect of random shocks? Management Science, 65(3):1096-1114, 2019.

Sanjit Dhami. The Foundations of Behavioral Economic Analysis. Oxford University Press, 2016.

Sanjit Dhami and Ali Al-Nowaihi. Why do people pay taxes? prospect theory versus expected utility theory. Journal of Economic Behavior \& Organization, 64(1):171-192, 2007. 
Nathalie Etchart-Vincent. Is probability weighting sensitive to the magnitude of consequences? an experimental investigation on losses. Journal of Risk and Uncertainty, 28(3):217-235, 2004.

Nathalie Etchart-Vincent. Probability weighting and the 'level'and 'spacing' of outcomes: An experimental study over losses. Journal of Risk and Uncertainty, 39(1):45-63, 2009a.

Nathalie Etchart-Vincent. The shape of the utility function under risk in the loss domain and the'ruinous losses' hypothesis: some experimental results. Economics Bulletin, 29(2): 1404-1413, 2009b.

Nathalie Etchart-Vincent and Olivier l'Haridon. Monetary incentives in the loss domain and behavior toward risk: An experimental comparison of three reward schemes including real losses. Journal of Risk and Uncertainty, 42(1):61-83, 2011.

Helga Fehr-Duda, Manuele De Gennaro, and Renate Schubert. Gender, financial risk, and probability weights. Theory and Decision, 60(2-3):283-313, 2006.

Paolo Ghirardato and Massimo Marinacci. Risk, ambiguity, and the separation of utility and beliefs. Mathematics of Operations Research, 26(4):864-890, 2001.

Itzhak Gilboa. Expected utility with purely subjective non-additive probabilities. Journal of Mathematical Economics, 16(1):65-88, 1987.

William M Goldstein and Hillel J Einhorn. Expression theory and the preference reversal phenomena. Psychological Review, 94(2):236, 1987.

Richard Gonzalez and George Wu. On the shape of the probability weighting function. Cognitive Psychology, 38(1):129-166, 1999.

Narges Hajimoladarvish. Very low probabilities in the loss domain. The Geneva Risk and Insurance Review, 42(1):41-58, 2017.

Glenn W Harrison and Elisabet E Rutström. Risk aversion in the laboratory. In Risk Aversion in Experiments, pages 41-196. Emerald Group Publishing Limited, 2008.

Glenn W Harrison and Elisabet E Rutström. Expected utility theory and prospect theory: One wedding and a decent funeral. Experimental Economics, 12(2):133-158, 2009.

John D Hey and Chris Orme. Investigating generalizations of expected utility theory using experimental data. Econometrica, 62(6):1291-1326, 1994.

John D Hey, Andrea Morone, and Ulrich Schmidt. Noise and bias in eliciting preferences. Journal of Risk and Uncertainty, 39(3):213-235, 2009.

Charles A Holt and Susan K Laury. Risk aversion and incentive effects. American Economic Review, 92(5):1644-1655, 2002.

Cathleen Johnson, Aurélien Baillon, Zhihua Li, Dennie van Dolder, and Peter P. Wakker. Prince: An improved method for measuring incentivized preferences. 2019.

Daniel Kahneman and Amos Tversky. Prospect theory: An analysis of decision under risk. Econometrica, 47(2):263-292, 1979.

Emmanuel Kemel and Sofiia Mun. An econometric estimation of prospect theory for natural ambiguity. Conference D-TEA 2020 on Prospect Theory, 2020. 
Veronika Köbberling and Peter P Wakker. An index of loss aversion. Journal of Economic Theory, 122(1):119-131, 2005.

Pamela K Lattimore, Joanna R Baker, and Ann D Witte. The influence of probability on risky choice: A parametric examination. Journal of Economic Behavior \&5 Organization, 17(3): 377-400, 1992.

Joost M E Pennings and Ale Smidts. The shape of utility functions and organizational behavior. Management Science, 49(9):1251-1263, 2003.

Thierry Post, Martijn J Van den Assem, Guido Baltussen, and Richard H Thaler. Deal or no deal? decision making under risk in a large-payoff game show. American Economic Review, 98(1):38-71, 2008.

John Quiggin. A theory of anticipated utility. Journal of Economic Behavior 8 Organization, $3(4): 323-343,1982$.

David Schmeidler. Subjective probability and expected utility without additivity. Econometrica, pages 571-587, 1989.

Chris Starmer. Developments in non-expected utility theory: The hunt for a descriptive theory of choice under risk. Journal of Economic Literature, 38(2):332-382, 2000.

Henry P Stott. Cumulative prospect theory's functional menagerie. Journal of Risk and Uncertainty, 32(2):101-130, 2006.

Tomomi Tanaka, Colin F Camerer, and Quang Nguyen. Risk and time preferences: Linking experimental and household survey data from vietnam. American Economic Review, 100(1): $557-571,2010$.

Sabrina M Tom, Craig R Fox, Christopher Trepel, and Russell A Poldrack. The neural basis of loss aversion in decision-making under risk. Science, 315(5811):515-518, 2007.

Amos Tversky and Daniel Kahneman. Advances in prospect theory: Cumulative representation of uncertainty. Journal of Risk and Uncertainty, 5(4):297-323, 1992.

Gijs Van De Kuilen and Peter P Wakker. The midweight method to measure attitudes toward risk and ambiguity. Management Science, 57(3):582-598, 2011.

Peter Wakker and Daniel Deneffe. Eliciting von neumann-morgenstern utilities when probabilities are distorted or unknown. Management Science, 42(8):1131-1150, 1996.

Peter P Wakker. Prospect Theory: For Risk and Ambiguity. Cambridge University Press, 2010.

Menahem E Yaari. The dual theory of choice under risk. Econometrica, 55(1):95-115, 1987. 


\title{
Appendix
}

\author{
A1-Empirical data \\ A1.1 - Data
}

a) Source

We use median data previously reported by TK92. They run a computerized experiment with 25 graduate students from Berkeley and Stanford with no particular training in decision theory. Each subject participates in three separate one-hour sessions organized several days apart, and receives $\$ 25$ for participation. We use all the median observations from non-mixed prospects (see their Table 3) as well as the first six median observations from mixed prospects (see their Table 6).

\section{b) Procedure}

The data are generated via the switching outcome procedure - in which indifference value is inferred through a list of equally spaced certain outcomes, ranging from the admissible maximum indifference value to the admissible minimum indifference value. Note that an alternative approach, the direct matching procedure - in which subjects are directly asked to provide their indifference values - tends to produce more inconsistencies (Bostic et al., 1990; Booij and Van de Kuilen, 2009). Internal consistency of the responses to each prospect is monitored by the computer software to reduce response errors.

c) Data for the first step

The outcomes are expressed in US dollars. In Table 3 of TK92, there are 28 median values of certainty equivalent for binary lotteries that involve 7 pairs of positive monetary outcomes $-(0,50),(0,100),(0,200),(0,400),(50,100),(50,150)$ and $(100,200)$, and 9 probabilities $0.01,0.05,0.1,0.25,0.5,0.75,0.9,0.95$ and 0.99 - of getting the higher outcome.

Also, in the same Table 3 of TK92 there are 28 median values of certainty equivalent for binary lotteries that involve 7 pairs of negative monetary outcomes - $(0,-50),(0,-100),(0$, $-200),(0,-400),(-50,-100),(-50,-150)$ and $(-100,-200)$, and the same list of 9 probabilities as in the gain domain. These probabilities are now associated to losing the higher outcome.

As required by our method, at least two certainty equivalents for each of the nine probabilities are available per domain so as to perform simultaneous measurement of utility function and probability weighting function in the full domain.

c) Data for the second step

The outcomes are expressed in US dollars. In table 6 of TK92, there are 6 indifferences that involves mixed prospects. The first four items consist in eliciting values of gains $x$ to make subjects indifferent between the mixed prospects $(x, y ; 0.5,0.5)$ and 0 . The values of $y$ are -25 , $-50,-100$ and -150 .

The two others indifferences consist in eliciting gains $\mathrm{x}$ that make subjects indifferent between two mixed prospects $(x, y ; 0.5,0.5)$ and $(z, w ; 0.5,0.5)$. The triplets $(y, z, w)$ take the values of either $(-50,50,-20)$ or $(-125,150,-50)$. Note that here the experimenter has no control on the maximum level of $x$ which may hinder the use of switching outcome procedure to find indifference value. For this reason, we make change in the third step of the method of ABL through equations (13) - (16). Also, our method is based on the comparisons of binary lotteries 
and sure outcomes. Hence, these data do not exactly fit our method. ${ }^{31}$ With the present data, we compute loss aversion for each of the six questions and take the median as estimated value to account for response error (following ABL). For the first four items, we compute loss aversion as follows:

$$
\begin{gathered}
\lambda=\frac{w^{+}(0.5)}{w^{-}(0.5)} \times \frac{x^{\alpha_{p}}}{(-y)^{\beta_{p}}} \\
\lambda=\frac{w^{+}(0.5)}{w^{-}(0.5)} \times \frac{1-\exp \left(-\alpha_{e} x\right)}{1-\exp \left(\beta_{e} y\right)} \times \frac{\beta_{e}}{\alpha_{e}}
\end{gathered}
$$

Formulas (17) and (18) correspond to power and exponential specification, respectively.

For the last two questions, we compute loss aversion as follows

$$
\begin{gathered}
\lambda=\frac{w^{+}(0.5)}{w^{-}(0.5)} \times \frac{z^{\alpha_{p}}-x^{\alpha_{p}}}{(-w)^{\beta_{p}}-(-y)^{\beta_{p}}} \\
\lambda=\frac{w^{+}(0.5)}{w^{-}(0.5)} \times \frac{\exp \left(-\alpha_{e} x\right)-\exp \left(-\alpha_{e} z\right)}{\exp \left(\beta_{e} y\right)-\exp \left(\beta_{e} w\right)} \times \frac{\beta_{e}}{\alpha_{e}}
\end{gathered}
$$

Formulas (19) and (20) correspond to the power and exponential specifications, respectively. Finally, following ABL, we compute the median loss aversion.

A1.2 - Our main semi-parametric measurements

Our main semi-parametric measurements are presented in tables 3 and 4 .

A1.3 - Semi-parametric results under identical probability weighting function and duality assumptions

Tables 5 and 6 present these results.

A1.4- Parametric measurements

TK92 used the following one-parameter (per domain) probability weighting function

$$
w^{i}(p)=\frac{p^{\left(\gamma \mathbf{1}_{(i=+)}+\theta \mathbf{1}_{(i=-)}\right)}}{\left[p^{\left(\gamma \mathbf{1}_{(i=+)}+\theta \mathbf{1}_{(i=-)}\right)}+(1-p)^{\left(\gamma \mathbf{1}_{(i=+)}+\theta \mathbf{1}_{(i=-)}\right)}\right]^{\frac{1}{\gamma \mathbf{1}_{(i=+)}+\theta \mathbf{1}_{(i=-)}}}}
$$

Note that this probability weighting function is for the full domain. Combining this with the power utility function (4) and using the same notations as in section 3 , we arrive at the following equations:

$\boldsymbol{c} \boldsymbol{e}_{l}=\left(\boldsymbol{D}_{l}^{+}-\boldsymbol{D}_{l}^{-}\right)\left[\left(\left|\boldsymbol{x}_{l}\right|^{\alpha_{p} D_{l}^{+}+\beta_{p} D_{l}^{-}}-\left|\boldsymbol{y}_{l}\right|^{\alpha_{p} D_{l}^{+}+\beta_{p} D_{l}^{-}}\right) \frac{p^{\left(\gamma D_{l}^{+}+\theta D_{l}^{-}\right)}}{\left(p^{\left(\gamma D_{l}^{+}+\theta D_{l}^{-}\right)}+(1-p)^{\left(\gamma D_{l}^{+}+\theta D_{l}^{-}\right)}\right)^{\frac{1}{\gamma D_{l}^{+}+\theta D_{l}^{-}}}}+\left|\boldsymbol{y}_{l}\right|^{\alpha_{p} D_{l}^{+}+\beta_{p} D_{l}^{-}}\right]^{\frac{1}{\alpha_{p} D_{l}^{+}+\beta_{p} D_{l}^{-}}}+\mathbf{e}_{l}$

$c e_{l}=\frac{\ln \left[\left(\exp \left(\left(\beta_{e} \boldsymbol{D}_{l}^{-}-\alpha_{e} \boldsymbol{D}_{l}^{+}\right) \boldsymbol{x}_{l}\right)-\exp \left(\left(\beta_{e} \boldsymbol{D}_{l}^{-}-\alpha_{e} \boldsymbol{D}_{l}^{+}\right) \boldsymbol{y}_{l}\right)\right) \frac{p^{\left(\gamma \boldsymbol{D}_{l}^{+}+\theta \boldsymbol{D}_{l}^{-}\right)}}{\left(p^{\left(\gamma \boldsymbol{D}_{l}^{+}+\theta \boldsymbol{D}_{l}^{-}\right)}+(1-p)^{\left(\gamma \boldsymbol{D}_{l}^{+}+\theta \boldsymbol{D}_{l}^{-}\right)}\right)^{\frac{1}{\boldsymbol{D}_{l}^{+}+\theta \boldsymbol{D}_{l}^{-}}}+\exp \left(\left(\beta_{e} \boldsymbol{D}_{l}^{-}-\alpha_{e} \boldsymbol{D}_{l}^{+}\right) \boldsymbol{y}_{l}\right)}\right]}{\beta_{e} \boldsymbol{D}_{l}^{-}-\alpha_{e} \boldsymbol{D}_{l}^{+}}+\mathbf{e}_{l}$

\footnotetext{
${ }^{31}$ Based on simulation exercises, we observe that these equations work well.
} 
Table 3: Results of the first step

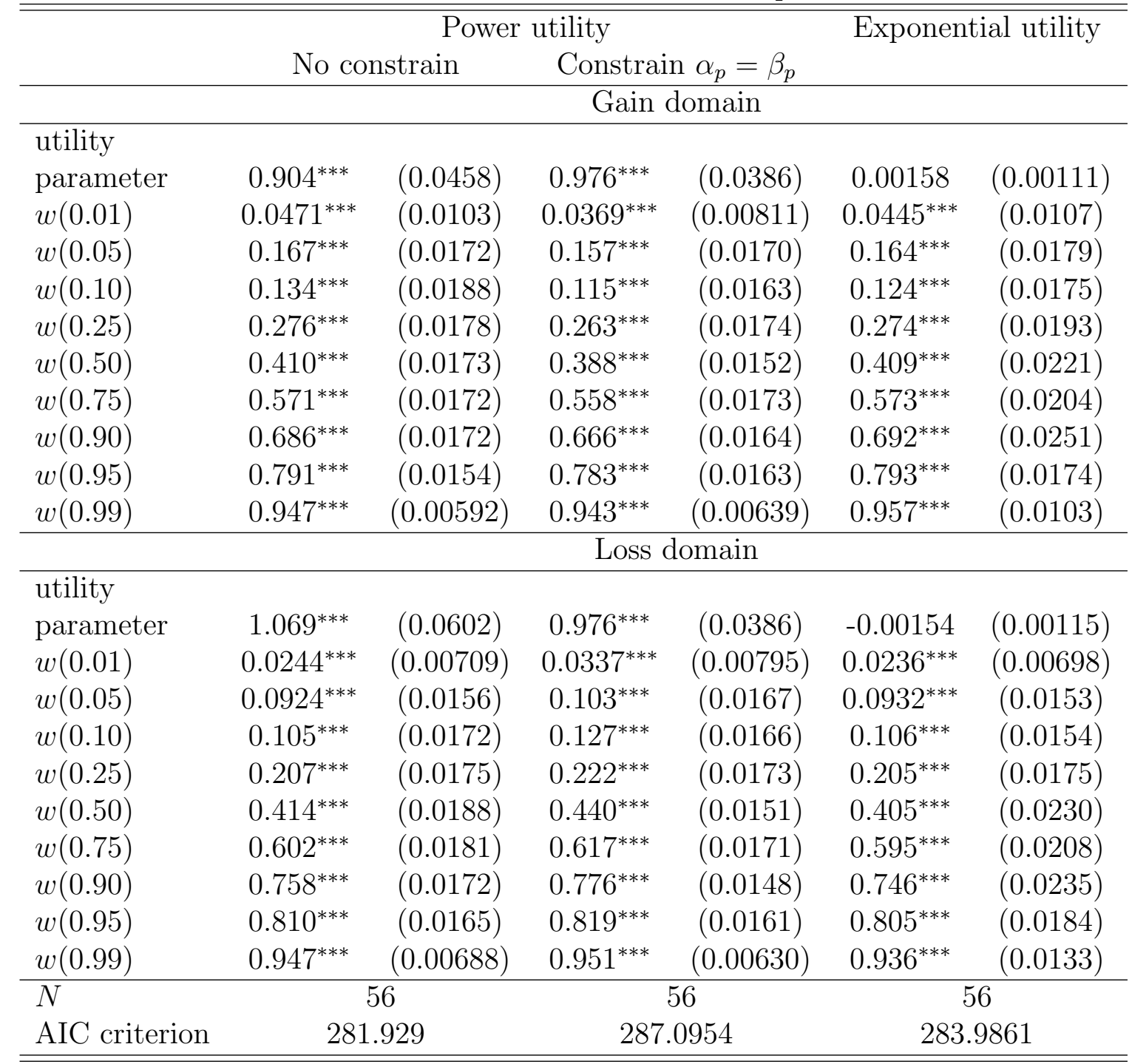

Standard errors in parentheses.

${ }^{*} p<0.1,{ }^{* *} p<0.05,{ }^{* * *} p<0.01$ to test the significance of coefficients.

Table 4: Results of the second step (Loss aversion)

\begin{tabular}{lccc}
\hline \hline Observations & \multicolumn{2}{c}{ Power utility } & Exponential utility \\
\hline \hline 1 & No constrain & Constrain $\alpha_{p}=\beta_{p}$ & \\
2 & 1.304 & 2.106 & 2.306 \\
3 & 0.981 & 1.751 & 1.815 \\
4 & 0.875 & 1.751 & 1.616 \\
5 & 0.762 & 1.622 & 1.355 \\
6 & 0.892 & 1.786 & 1.741 \\
\hline Median & 0.739 & 1.735 & 1.247 \\
\hline \hline
\end{tabular}

This allows us to elicit in full domain utility and probability weighting function parameters of TK92.

Once the parameters of utility function and probability weighting function are obtained 
Table 5: Results under identical probability weighting assumption

\begin{tabular}{lcccc}
\hline \hline & \multicolumn{2}{c}{ Power utility } & \multicolumn{2}{c}{ Exponential utility } \\
\hline Utility parameter (Gains) & $0.904^{* * *}$ & $(0.0504)$ & 0.00132 & $(0.00105)$ \\
Utility parameter (Losses) & $1.053^{* * *}$ & $(0.0586)$ & -0.00127 & $(0.00107)$ \\
$w^{+}(0.01)=w^{-}(0.01)$ & $0.0326^{* * *}$ & $(0.00839)$ & $0.0301^{* * *}$ & $(0.00771)$ \\
$w^{+}(0.05)=w^{-}(0.05)$ & $0.128^{* * *}$ & $(0.0164)$ & $0.125^{* * *}$ & $(0.0153)$ \\
$w^{+}(0.10)=w^{-}(0.10)$ & $0.119^{* * *}$ & $(0.0177)$ & $0.114^{* * *}$ & $(0.0150)$ \\
$w^{+}(0.25)=w^{-}(0.25)$ & $0.242^{* * *}$ & $(0.0174)$ & $0.237^{* * *}$ & $(0.0170)$ \\
$w^{+}(0.50)=w^{-}(0.50)$ & $0.414^{* * *}$ & $(0.0177)$ & $0.408^{* * *}$ & $(0.0205)$ \\
$w^{+}(0.75)=w^{-}(0.75)$ & $0.587^{* * *}$ & $(0.0173)$ & $0.584^{* * *}$ & $(0.0189)$ \\
$w^{+}(0.90)=w^{-}(0.90)$ & $0.720^{* * *}$ & $(0.0169)$ & $0.715^{* * *}$ & $(0.0223)$ \\
$w^{+}(0.95)=w^{-}(0.95)$ & $0.801^{* * *}$ & $(0.0156)$ & $0.799^{* * *}$ & $(0.0164)$ \\
$w^{+}(0.99)=w^{-}(0.99)$ & $0.947^{* * *}$ & $(0.00621)$ & $0.950^{* * *}$ & $(0.0106)$ \\
\hline$N$ & \multicolumn{2}{c}{56} & \multicolumn{2}{c}{56} \\
AIC criterion & 313.400 & 308.596 \\
\hline \hline
\end{tabular}

Standard errors in parentheses

${ }^{*} p<0.1,{ }^{* *} p<0.05,{ }^{* * *} p<0.01$

Table 6: Results under duality assumption $w^{+}(p)=1-w^{-}(1-p)$

\begin{tabular}{lcccc}
\hline \hline & \multicolumn{3}{c}{ Power utility } & \multicolumn{2}{c}{ Exponential utility } \\
\hline Utility parameter (Gains) & $0.738^{* * *}$ & $(0.0525)$ & $0.00771^{* * *}$ & $(0.00165)$ \\
Utility parameter (Losses) & $0.754^{* * *}$ & $(0.0529)$ & $0.00270^{*}$ & $(0.00155)$ \\
$w^{+}(0.01)=1-w^{-}(0.99)$ & $0.0431^{* * *}$ & $(0.00913)$ & $0.0330^{* * *}$ & $(0.0122)$ \\
$\left.w^{+}(0.05)=1-w^{-} 0.95\right)$ & $0.170^{* * *}$ & $(0.0221)$ & $0.178^{* * *}$ & $(0.0237)$ \\
$\left.w^{+}(0.10)=1-w^{-} 0.90\right)$ & $0.182^{* * *}$ & $(0.0212)$ & $0.187^{* * *}$ & $(0.0272)$ \\
$\left.w^{+}(0.25)=1-w^{-} 0.75\right)$ & $0.327^{* * *}$ & $(0.0232)$ & $0.347^{* * *}$ & $(0.0268)$ \\
$w^{+}(0.50)=1-w^{-}(0.50)$ & $0.478^{* * *}$ & $(0.0218)$ & $0.516^{* * *}$ & $(0.0299)$ \\
$w^{+}(0.75)=1-w^{-}(0.25)$ & $0.662^{* * *}$ & $(0.0232)$ & $0.693^{* * *}$ & $(0.0253)$ \\
$\left.w^{+}(0.90)=1-w^{-} 0.10\right)$ & $0.755^{* * *}$ & $(0.0229)$ & $0.817^{* * *}$ & $(0.0269)$ \\
$w^{+}(0.95)=1-w^{-}(0.05)$ & $0.834^{* * *}$ & $(0.0219)$ & $0.856^{* * *}$ & $(0.0204)$ \\
$w^{+}(0.99)=1-w^{-}(0.01)$ & $0.953^{* * *}$ & $(0.00907)$ & $0.989^{* * *}$ & $(0.00512)$ \\
\hline$N$ & \multicolumn{3}{c}{56} & \multicolumn{2}{c}{56} \\
AIC criterion & \multicolumn{3}{c}{353.9371} & \multicolumn{3}{c}{} \\
\hline \hline
\end{tabular}

Standard errors in parentheses

${ }^{*} p<0.1,{ }^{* *} p<0.05,{ }^{* * *} p<0.01$ to test the significance of coefficients.

with equation (22) and (23), we estimate loss aversion as described in equations (17) - (20).

Table 7 presents the results of the first step that measures simultaneously the utility functions and probability weighting functions in the full domain. Tables 8 and 9 summarize the estimates of loss aversion using parameters of the probability weighting and utility functions from the first step as inputs.

A1.5-Parametric results using two-parameter weighting functions

The basic power utility function (4) and the following probability weighting function of Goldstein and Einhorn (1987) and Lattimore et al. (1992) are used to perform parametric measurements. 
Table 7: Results of the first step

\begin{tabular}{|c|c|c|c|c|c|c|}
\hline & \multicolumn{4}{|c|}{ Power utility } & \multirow{2}{*}{\multicolumn{2}{|c|}{ Exponential utility }} \\
\hline & \multicolumn{2}{|c|}{ No constrain } & \multicolumn{2}{|c|}{ Constrain $\alpha_{p}=\beta_{p}$} & & \\
\hline \multicolumn{7}{|c|}{ Gain domain } \\
\hline Utility Parameter & $0.839^{* * *}$ & $(0.0285)$ & $0.872^{* * *}$ & $(0.0209)$ & $0.00168^{* *}$ & $(0.000652)$ \\
\hline$\gamma$ & $0.643^{* * *}$ & $(0.0125)$ & $0.635^{* * *}$ & $(0.0115)$ & $0.649^{* * *}$ & $(0.0193)$ \\
\hline \multicolumn{7}{|c|}{ Loss domain } \\
\hline Utility Parameter & $0.906^{* * *}$ & $(0.0298)$ & $0.872^{* * *}$ & $(0.0209)$ & $0.00155^{* *}$ & $(0.000698)$ \\
\hline$\theta$ & $0.704^{* * *}$ & $(0.0155)$ & $0.713^{* * *}$ & $(0.0154)$ & $0.721^{* * *}$ & $(0.0234)$ \\
\hline$N$ & \multicolumn{2}{|c|}{56} & \multicolumn{2}{|c|}{56} & \multicolumn{2}{|r|}{56} \\
\hline AIC criterion & \multicolumn{2}{|c|}{318.248} & \multicolumn{2}{|c|}{318.997} & \multicolumn{2}{|c|}{337.134} \\
\hline
\end{tabular}

Standard errors in parentheses

${ }^{*} p<0.1,{ }^{* *} p<0.05,{ }^{* * *} p<0.0101$ to test the significance of coefficients

Table 8: Results of the second step (Loss aversion)

\begin{tabular}{lccc}
\hline \hline Observations & \multicolumn{2}{c}{ Power utility } & Exponential utility \\
\hline \hline 1 & No constrain & Constrain $\alpha_{p}=\beta_{p}$ & \\
2 & 1.618 & 2.040 & 2.234 \\
3 & 1.318 & 1.730 & 1.824 \\
4 & 1.258 & 1.730 & 1.747 \\
5 & 1.146 & 1.615 & 1.577 \\
6 & 1.253 & 1.738 & 1.800 \\
\hline Median & 1.127 & 1.668 & 1.496 \\
\hline \hline
\end{tabular}

$$
w^{i}(p)=\frac{\left(b \mathbf{1}_{(i=+)}+d \mathbf{1}_{(i=-)}\right) p^{\left(a \mathbf{1}_{(i=+)}+c \mathbf{1}_{(i=-)}\right)}}{\left(b \mathbf{1}_{(i=+)}+d \mathbf{1}_{(i=-)}\right) p^{\left(a \mathbf{1}_{(i=+)}+c \mathbf{1}_{(i=-)}\right)}+(1-p)^{\left(a \mathbf{1}_{(i=+)}+c \mathbf{1}_{(i=-)}\right)}}
$$

Combining this with the power utility function (4) and using the same notations as in section 3 , we have the following equation that allows to elicit in full domain utility and probability weighting function parameters:

$c e_{l}=\left(\boldsymbol{D}_{l}^{+}-\boldsymbol{D}_{l}^{-}\right)\left[\left(\left|\boldsymbol{x}_{l}\right|^{\alpha_{p} D_{l}^{+}+\beta_{p} D_{l}^{-}}-\left|\boldsymbol{y}_{l}\right|^{\alpha_{p} D_{l}^{+}+\beta_{p} D_{l}^{-}}\right) \frac{\left(b D_{l}^{+}+d D_{l}^{-}\right) p^{\left(a D_{l}^{+}+c D_{l}^{-}\right)}}{\left(b D_{l}^{+}+d \boldsymbol{D}_{l}^{-}\right) p^{\left(a D_{l}^{+}+c D_{l}^{-}\right)}+(1-p)^{\left(a D_{l}^{+}+c D_{l}^{-}\right)}}+\left|y_{l}\right|^{\alpha_{p} D_{l}^{+}+\beta_{p} D_{l}^{-}}\right]^{\frac{1}{\alpha_{p} D_{l}^{+}+\beta_{p} D_{l}^{-}}}+\mathbf{e}_{l}$

Table 9 presents the estimates of equation (25). The elicited utility function is concave in both domains. The estimated two-parameter probability weighting functions of Lattimore et al. (1992) are less elevated than the estimated one-parameter probability weighting functions of TK92. These parametric results on utility curvature and probability weighting function using the two-parameter probability weighting functions of Lattimore et al. (1992) are in line with the results of Fehr-Duda et al. (2006) as well as with our semi-parametric measurements.

\section{A2- Application to data of GW99}

In this part, we replicate the non-parametric estimates of GW99 using our semi-parametric method. 
Table 9: Results of the first step

\begin{tabular}{llc}
\hline \hline \multicolumn{1}{c}{ Variable } & Coefficient & (Std. Err.) \\
\hline$\alpha_{p}$ & Gain domain \\
$a$ & $0.907^{* *}$ & $(0.056)$ \\
$b$ & $0.620^{* *}$ & $(0.019)$ \\
\hline \multicolumn{3}{c}{ Loss domain } \\
\hline$\beta_{p}$ & $1.053^{* *}$ & $(0.057)$ \\
$c$ & $0.673^{* *}$ & $(0.068)$ \\
$d$ & $0.708^{* *}$ & $(0.025)$ \\
\hline \multicolumn{3}{c}{56} \\
\hline $\mathrm{N}$ & \multicolumn{3}{c}{316.223} \\
AIC criterion & \multicolumn{3}{c}{$* 5 \% \quad * *: 1 \%$}
\end{tabular}

\section{A2.1-Data}

\section{Procedure}

We use median data previously reported by GW99. They run a computerized experiment with 10 graduate students in psychology. Like TK92, GW99 use the switching outcome procedure.

\section{Lotteries}

All outcomes are expressed in US dollars. Outcomes 0, 25, 50, 75, 100, 150, 200, 400 and 800 are used to form 15 pairs of positive monetary outcomes: $(0,25),(0,50),(0,75),(0,100)$, $(0,150),(0,200),(0,400),(0,800),(25,50),(50,75),(50,100),(50,150),(100,150),(100$, $200)$ and $(150,200)$. In Table 1 in GW99, there are 165 median values of certainty equivalents for binary lotteries that involve these 15 pairs of positive monetary outcomes and 11 probabilities - 0.01, 0.05, 0.1, 0.25, 0.4, 0.5, 0.6, 0.75, 0.9, 0.95 and 0.99 - of getting the higher outcome.

\section{A2.2- Semi-parametric measurements}

Here, we present the estimates from our semi-parametric method under power and exponential utility functions applied to the median data for GW99. The data only covers the gain domain. With the same notation as in section 3, equation (8) collapses to:

$$
\boldsymbol{c e}_{l}=u^{-1}\left[\left(u\left(\boldsymbol{x}_{l}\right)-u\left(\boldsymbol{y}_{l}\right)\right) \sum_{k=1}^{K} \delta_{k}^{+} \boldsymbol{I}_{l}^{k}+u\left(\boldsymbol{y}_{l}\right)\right]+\mathbf{e}_{l}
$$

where $l$ is the $l^{\text {th }}$ line in $\boldsymbol{c e}, \boldsymbol{x}$ and $\boldsymbol{y}$ and $u^{-1}$ is the inverse of utility function in gain domain. In (26), probability weights can be derived as follows:

$$
w^{+}\left(p_{k}\right)=\delta_{k}^{+} \text {for } k=1, . ., K
$$

We can then derive the certainty equivalent equation (26) for both power (28) and exponential (29) utility functions as follows: 


$$
\begin{gathered}
\boldsymbol{c} \boldsymbol{e}_{l}=\left[\left(\boldsymbol{x}_{l}^{\alpha_{p}}-\boldsymbol{y}_{l}^{\alpha_{p}}\right) \sum_{k=1}^{K} \delta_{k}^{+} \boldsymbol{I}_{l}^{k}+\boldsymbol{y}_{l}^{\alpha_{p}}\right]^{\frac{1}{\alpha_{p}}}+\mathbf{e}_{l} \\
\boldsymbol{c e}_{l}=-\frac{1}{\alpha_{e}} \ln \left[\left(\exp \left(-\alpha_{e} \boldsymbol{x}_{l}\right)-\exp \left(-\alpha_{e} \boldsymbol{y}_{l}\right)\right) \sum_{k=1}^{K} \delta_{k}^{+} \boldsymbol{I}_{l}^{k}+\exp \left(-\alpha_{e} \boldsymbol{y}_{l}\right)\right]+\mathbf{e}_{l}
\end{gathered}
$$

Table 10 presents the estimates of equation (28) and (29). The AIC is lower for power utility function $(\mathrm{AIC}=1208.047)$ than for exponential utility function ( $\mathrm{AIC}=1252.64)$. Hence, power utility function has a better fit than the exponential utility function.

Table 10: Semi-parametric estimations on the median data of GW99

\begin{tabular}{lcccc}
\hline \hline & \multicolumn{2}{c}{ Power utility } & \multicolumn{2}{c}{ Exponential utility } \\
\hline Utility parameter & $0.511^{* * *}$ & $(0.0323)$ & $0.00101^{* * *}$ & $(0.000172)$ \\
$w^{+}(0.01)$ & $0.143^{* * *}$ & $(0.0316)$ & $0.0365^{* *}$ & $(0.0145)$ \\
$w^{+}(0.05)$ & $0.223^{* * *}$ & $(0.0274)$ & $0.0782^{* * *}$ & $(0.0145)$ \\
$w^{+}(0.10)$ & $0.227^{* * *}$ & $(0.0273)$ & $0.0816^{* * *}$ & $(0.0145)$ \\
$w^{+}(0.25)$ & $0.338^{* * *}$ & $(0.0258)$ & $0.165^{* * *}$ & $(0.0151)$ \\
$w^{+}(0.40)$ & $0.355^{* * *}$ & $(0.0257)$ & $0.181^{* * *}$ & $(0.0152)$ \\
$w^{+}(0.50)$ & $0.349^{* * *}$ & $(0.0257)$ & $0.175^{* * *}$ & $(0.0151)$ \\
$w^{+}(0.60)$ & $0.485^{* * *}$ & $(0.0237)$ & $0.310^{* * *}$ & $(0.0165)$ \\
$w^{+}(0.75)$ & $0.505^{* * *}$ & $(0.0232)$ & $0.336^{* * *}$ & $(0.0168)$ \\
$w^{+}(0.90)$ & $0.700^{* * *}$ & $(0.0170)$ & $0.583^{* * *}$ & $(0.0173)$ \\
$w^{+}(0.95)$ & $0.750^{* * *}(0.0149)$ & $0.653^{* * *}$ & $(0.0165)$ \\
$w^{+}(0.99)$ & $0.813^{* * *}(0.0121)$ & $0.739^{* * *}$ & $(0.0150)$ \\
\hline$N$ & \multicolumn{3}{c}{165} \\
AIC criterion & \multicolumn{2}{c}{1208.047} & \multicolumn{2}{c}{1252.641} \\
\hline \hline
\end{tabular}

Standard errors in parentheses

${ }^{*} p<0.1,{ }^{* *} p<0.05,{ }^{* * *} p<0.01$ to test the significance of coefficients.

\section{Utility function}

To compare our estimated utility function with that of GW99, we normalize the two utility functions on the same scale, so that $u(\$ 0)=0$ and $u(\$ 800)=1$. Figure 2 plots the two utility functions. We remark that the $95 \%$ confidence interval of the utility function provided by our method contains the estimated utility values of GW99. Hence, our utility function is not significantly different from that of GW99. We find $\alpha_{p}=0.511$ and this value is significantly different from $1(p-$ value $<0.001)$. This means that the utility is concave in gain domain.

\section{Probability weighting function}

Figure 3 displays the elicited probability weights based on our semi-parametric method along with the probability weights from GW99. As for the utility function, we found that the $95 \%$ confidence intervals of probability weights provided by our method contain the estimated probability weights of GW99. Hence, our probability weights are not significantly different from those of GW99. The probability weighting function from our semi-parametric method is inverse-S-shaped (concave, then convex) and crosses the $45^{\circ}$ line between $p=0.25$ and $p=0.4$. 
Figure 2: Semi-parametric measurement of utility function on median data of GW99

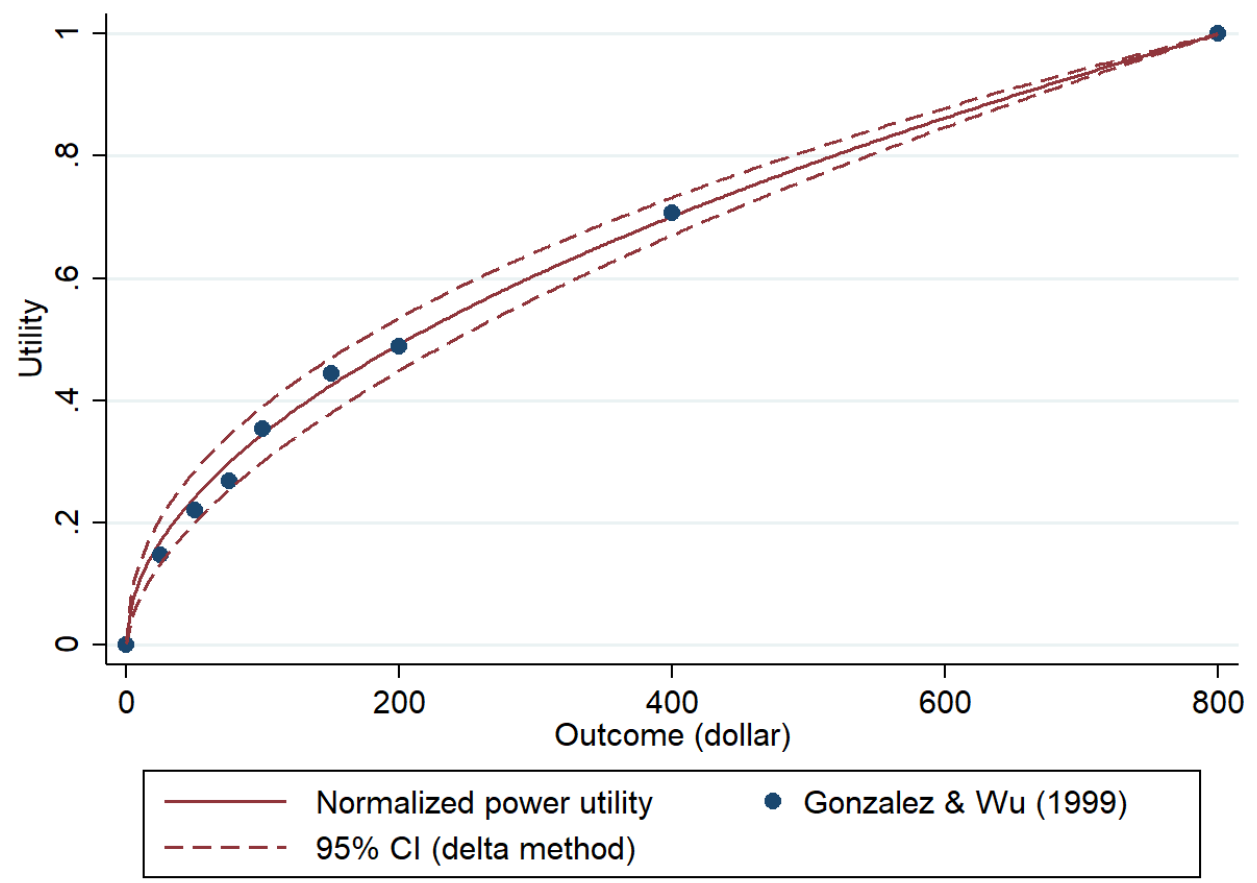

Figure 3: Semi-parametric measurement of probability weighting function on median data of GW99

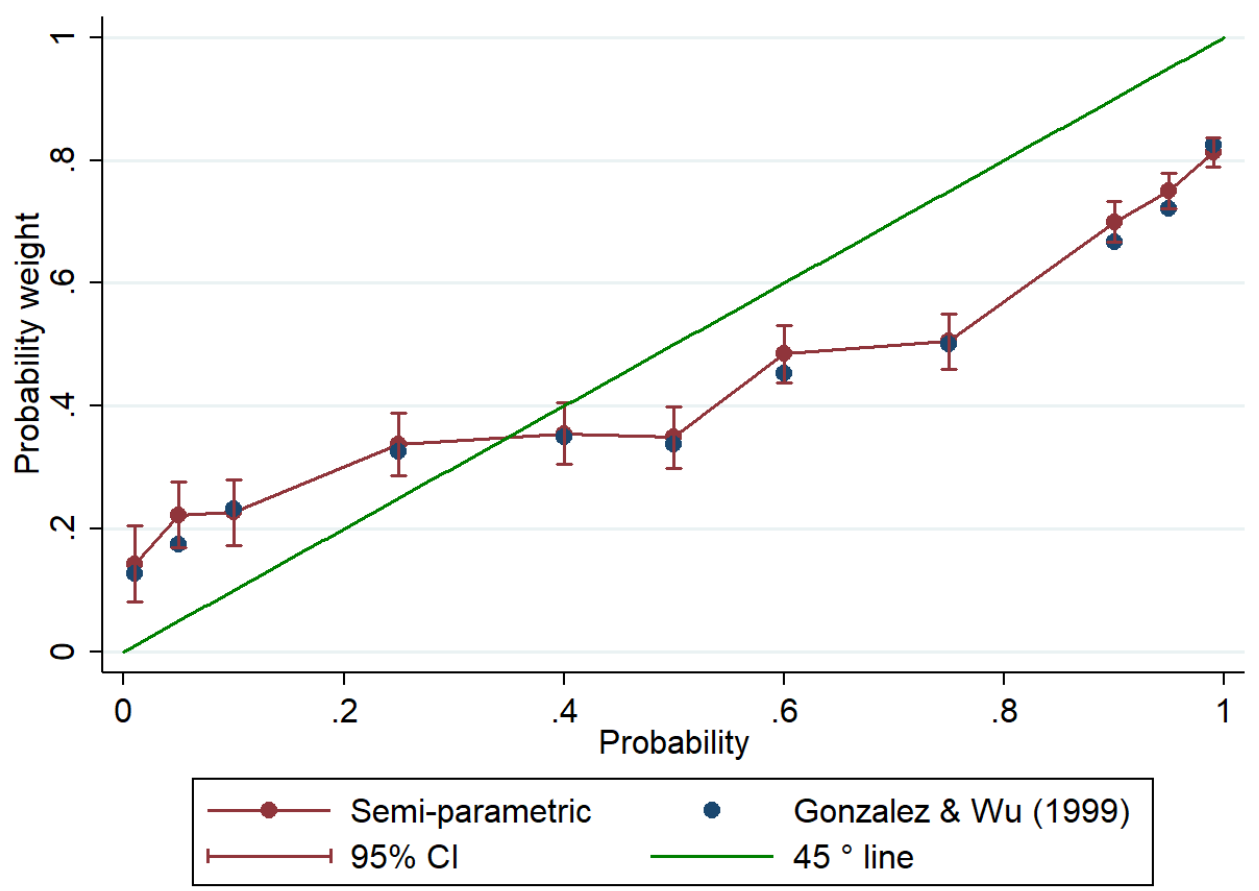

